\title{
Dynamical distortions of structural signatures in molecular high-order harmonic spectroscopy
}

\author{
Marie Labeye, ${ }^{1}$ François Risoud, ${ }^{1}$ Camille Lévêque,,${ }^{1,2, *}$ Jérémie Caillat, ${ }^{1}$ Alfred Maquet, ${ }^{1}$ \\ Tahir Shaaran, ${ }^{3}$ Pascal Salières, ${ }^{3}$ and Richard Taïeb ${ }^{1}$ \\ ${ }^{1}$ Sorbonne Université, CNRS, Laboratoire de Chimie Physique-Matière et Rayonnement, 75005 Paris, France \\ ${ }^{2}$ Theoretische Chemie, Physikalisch-Chemisches Institut, Universität Heidelberg, Im Neuenheimer Feld 229, D-69120 Heidelberg, Germany \\ ${ }^{3}$ LIDYL, CEA, CNRS, Université Paris-Saclay, CEA-Saclay, 91191 Gif-sur-Yvette, France
}

(Received 16 November 2018; published 11 January 2019)

\begin{abstract}
We study the signature of two-center interferences in molecular high-order harmonic spectra, with an emphasis on the spectral phase. With the help of both ab initio computations based on the time-dependent Schrödinger equation and the molecular strong-field approximation (SFA) as developed by Chirilă et al. [Phys. Rev. A 73, 023410 (2006)] and Faria [Phys. Rev. A 76, 043407 (2007)], we observe that the phase behavior is radically different for the short and the long trajectory contributions. By means of Taylor expansions of the molecular SFA, we link this effect to the dynamics of the electron in the continuum. More precisely, we find that the value of the electric field at recombination time plays a crucial role in the shape of the destructive interference phase jump.
\end{abstract}

DOI: 10.1103/PhysRevA.99.013412

\section{INTRODUCTION}

Over the past few decades, high-order harmonic generation (HHG) has led to an increasing interest in the field of strong laser physics with the development of attosecond science $[1,2]$. Two main classes of experiments have emerged in this context. First, HHG is a robust tool to generate single or trains of attosecond pulses of coherent extreme ultraviolet (XUV) radiation [3-10], which can be used to perform electronic or nuclear pump-probe spectroscopy on the attosecond scale with angstrom resolution [11-16]. Second, HHG can be employed as a self-probing tool to monitor attosecond dynamics in the generating medium [17-23], e.g., to implement tomography of molecular orbitals [24-26]. References $[27,28]$ provide a comprehensive review on the self-probing applications of HHG.

Most of these studies showed that the intensity and the phase of the generated harmonics encode structural and dynamical information. While the measurement of their intensity, i.e., counting the photon number for each wavelength, is rather straightforward, obtaining their phases was more difficult. At present, several experimental techniques based on interferometry have been mastered to access the harmonic phase, such as RABBIT (reconstruction of attosecond beating by interference of two-photon transition) [29,30], streaking [31,32], TSOIN (two-source optical interferometry) [33-35], PROOF (phase retrieval by omega oscillation filtering) [36], MAMMOTH (mixed approaches for the measurement of the total harmonic phases), CHASSEUR (combined harmonic

\footnotetext{
*Present address: Wolfgang Pauli Institute c/o Faculty of Mathematics, University of Vienna, Oskar-Morgenstern Platz 1, A-1090 Vienna, Austria and Vienna Center for Quantum Science and Technology, Atominstitut, TU Wien, Stadionallee 2, A-1020 Vienna, Austria.
}

attosecond spectroscopy by two-source euv interferometry and rabbit) [37], or other approaches [38,39]. These measures of the harmonic phase are crucial to study, e.g., the dynamics of autoionizing states or Fano resonances [12,37,40-47], interferences between different ionization paths [33,48-50], and two-center interferences [34,51-57].

To picture the phenomenon of HHG, the well-known threestep model is invoked [58,59]: (i) under the influence of a strong laser field, an electron is first pulled out from the atom or molecule, then (ii) scattered in the continuum and driven back to the parent ion, where (iii) it finally may recombine into its initial state, releasing radiatively its accumulated kinetic energy. The emitted photons constitute the harmonic spectrum. In this model, the electron in the continuum is considered as a classical particle and thus its motion is characterized by trajectories. Within the strong-field approximation (SFA) [60], which is a fully quantum theory, the notion of electron trajectories (or quantum orbits) in the continuum arises when searching saddle-point solutions in the equations leading to the emitted dipole [61-63], thus validating the three-step picture. Each harmonic of the spectrum is mainly emitted by two possible trajectories of the recolliding electron: the short and the long ones. Both lead to the same kinetic energy but with different times at which the electron recollides with the parent ion. The long trajectories correspond to electrons released before and recolliding after the short ones. Within a quantum theory, they interfere and shape the total spectrum with constructive and destructive patterns.

In the case of molecules, one may envision that electrons ionized from one atomic center and recombining to another will form additional classes of trajectories. Therefore, the structure of the system is encoded through additional interferences in the spectrum, which are reminiscent of Young's two-slit phenomenon in diatomics $[64,65]$.

In a standard SFA saddle-point approach [66-68], the structure of the molecule is usually introduced via the tran- 
sition matrix elements in steps 1 and 3, whereas the electron dynamics, i.e., step 2, remains atomic. Within this approach, destructive interferences are observed in the harmonic spectrum of diatomic molecules that correspond to the zeros of the recombination dipole matrix element. This results in a discontinuous phase jump of exactly $\pm \pi \operatorname{rad}$ in the theoretical spectra.

However, this behavior does not compare well with experimental data [51,53] nor with ab initio computations based on the exact resolution of the time-dependent Schrödinger equation (TDSE) $[64,65,69]$. The phase jumps at the destructive interferences are found to be far from sharp: they are smoothed, cover several harmonics and span less than $\pi \mathrm{rad}$.

Ten years ago, a method accounting for the effect of the molecular structure on the continuum electron dynamics in SFA computations, called molecular SFA, was proposed in [70] and closely investigated in [71]. It relies on the separation of the dipole into four terms, each one exhibiting an additional phase incorporated into the electronic action. The saddle-point approximation is carried out for each term and leads to four groups of possible electron trajectories (with their subsets of short and long paths). They correspond to the ionization from one center and the recollision to either the same center or the other one. To our knowledge, the shape of the phase jumps in such calculations has not been studied or addressed except in Ref. [55], where similar computations have been performed in the specific case of aligned polar $\mathrm{CO}$ molecules. Interestingly, the phase jumps are smoothed, which we will confirm with our simulations.

Other models based on the computation of SFA with fielddistorted orbitals also display smoothed phase jumps [56,57]. These approaches rely on an adiabatic dressing of the ground state by the instantaneous electric field at recombination. This modifies the shape of the corresponding dipole, and thus the shape of the spectrum. An important feature of this approach is that, if the recombination occurs at a time when the electric field is zero, then the recombination dipole is no longer modified by the dressing, and one recovers a sharp minimum in the spectrum.

We propose here a complete examination of the twocenter interferences in homonuclear diatomic molecular models, beyond the smoothing of the structural phase jumps. The motivation for this study arose when investigating HHG amplitude and phase for diatomic molecules as a function of the internuclear distance within $a b$ initio computations based on the TDSE. We observed very different behaviors between the phase jumps when discriminating the short and the long trajectories, in apparent relation with the electric field at the recombination. To obtain physical insight with an analytical theory, we compared with the results of molecular SFA [70,71] and found that they reproduce the $a b$ initio observations. Finally, we performed additional developments of the molecular SFA based on Taylor expansions, and they fully explain the shape of the phase jump in relation to the electric field at the recombination time.

This paper gives a full account of our results following our preliminary studies presented in [72]. It is organized as follows. In Sec. II we introduce the models we used. First, we expose the $a b$ initio computations we performed on a one-dimensional (1D) molecular model by solving the TDSE.
Second, we recall the basic equations of atomic SFA and molecular SFA and expose our further developments based on Taylor expansions of the molecular SFA. The ab initio computations will serve as the reference to compare with the SFA computations. In Sec. III we display the results of the ab initio and the molecular SFA computations in the case of a model $\mathrm{H}_{2}$. In Sec. IV we provide explanations of the behavior of the phase jumps with the examination of the Taylor expansions of the molecular SFA. We finally summarize our work in Sec. V and we will draw a qualitative parallel between the fielddistorted orbitals computations [56,57] and our developments. In this paper, all the equations are expressed in atomic units (a.u.).

\section{METHODS}

\section{A. Ab initio computations}

We consider a diatomic molecule subjected to a lowfrequency strong laser field with a 1D model within the single-active electron and fixed nuclei approximation [73], by solving numerically the TDSE. The computational efficiency of these simulations allows us to perform an extensive study of the problem. This approach serves as an exact reference illustrating the validity of our revisited molecular SFA and its interpretation presented hereafter.

We use a double-well potential $V(x)$ as in [74], where the interaction between the electron and each atomic center is modeled with a regularized Coulomb potential:

$$
V(x)=-\frac{1 / 2}{\sqrt{\left(x-\frac{R}{2}\right)^{2}+a^{2}}}-\frac{1 / 2}{\sqrt{\left(x+\frac{R}{2}\right)^{2}+a^{2}}} .
$$

Here $R$ is the internuclear distance, $a$ is the regularization parameter, and $x$ denotes the electron coordinate along the laser polarization $\mathbf{e}_{x}$. First, we will examine the two-center interference condition as a function of $R$, keeping fixed the ionization potential $I_{\mathrm{p}}$, thus adjusting the regularization parameter $a$ for each value of $R$. We have chosen the one of $\mathrm{H}_{2}: I_{\mathrm{p}}=0.567$ a.u. $=15.43 \mathrm{eV}$ [75]. Second, we will use this model for the specific case of $\mathrm{H}_{2}$ at equilibrium distance, where $R=1.4$ a.u. with $a=1.1339$ a.u.. The TDSE is solved for an electron in the ground state interacting with a two-cycle oscillating electric field $\mathbf{E}(t)=E_{\mathrm{L}} f(t) \sin (\omega t) \mathbf{e}_{x}$, where

$$
f(t)= \begin{cases}\sin ^{2}\left(\frac{\omega}{4} t\right) & \text { if } t \in[0,4 \pi / \omega], \\ 0 & \text { elsewhere. }\end{cases}
$$

Here $E_{\mathrm{L}}$ denotes the amplitude. Note that for such short pulses, the maximum of the electric field $E_{\max } \simeq 0.87 E_{\mathrm{L}}$ is substantially smaller than the amplitude $E_{\mathrm{L}}$. To be more consistent with the SFA computations, we define the peak intensity of our pulse as the square of $E_{\max }$, giving approximately $I_{\mathrm{L}} \simeq \frac{1}{2} \epsilon_{0} c E_{\mathrm{L}}^{2} / 1.322$, with $c$ the speed of light and $\epsilon_{0}$ the vacuum permittivity. We have set the frequency $\omega$ to 0.057 a.u., corresponding to a Ti:sapphire laser of $800 \mathrm{~nm}$ wavelength. With such a short IR pulse, the generated spectrum does not exhibit the usual harmonic comb structure: it is continuous through the entire plateau region. 
Within this model, the TDSE for the electronic wave function $\Psi(x, t)$ reads

$$
i \frac{\partial}{\partial t} \Psi(x, t)=\left[-\frac{1}{2} \frac{\partial^{2}}{\partial x^{2}}+V(x)+x E(t)\right] \Psi(x, t) .
$$

The HHG spectrum is proportional to the Fourier transform of the time-dependent dipole $\mathcal{D}(t)$, which is computed as the expectation value of the acceleration at each propagation time step [76]. While interest is often focused on the harmonic intensity, we focus on its phase as it encodes the dynamic of the system. The dominant contribution to the HHG phase arises from the intrinsic electron dynamics in the continuum (step 2). It is quadratic in energy and evolves rapidly [8,77]. Thus it might hide the small system-specific signatures in which we are interested. However, the dynamics of the electron freed in the continuum is common to all molecules or atoms of the same ionization potential in the same laser conditions. Computations of the HHG spectra from a reference atom with the same ionization potential are therefore used to remove this "universal" quadratic phase by subtracting it from the molecular HHG phase [8]. Our auxiliary atom is modeled by a single regularized Coulomb potential:

$$
V(x)=-\frac{1}{\sqrt{x^{2}+b^{2}}},
$$

for which we solve the TDSE with the same laser pulse parameters. Here $b=1.2195$ a.u. such that the ionization potential is again 0.567 a.u.

As the computed spectra contain contributions from both the short and long trajectories, we can use their different spatial properties to separate them and sort out their distinct signatures in the overall HHG phase. Classical computations show that the excursion of the electron trajectories in the continuum is characterized by a distance $x_{0}=E_{\mathrm{L}} / \omega^{2}$ [58]. The short trajectories never go beyond $x_{0}$ while the long trajectories always cross this value. Therefore, we first solved the TDSE with a numerical absorber [78] placed at $x_{0}$, which eliminates the wave-function components that go further away, corresponding classically to the long trajectories. As a consequence, the surviving part of the wave function is that of the short trajectories, and we can compute the corresponding dipole $\mathcal{D}_{s}(t)$. The solution of the same TDSE without the absorber at $x_{0}$ leads to the full dipole $\mathcal{D}(t)$. Thence, to recover the long trajectory contribution $\mathcal{D}_{l}(t)$, we subtract $\mathcal{D}_{s}(t)$ from $\mathcal{D}(t)$. One should note that this absorber artificially increases the plateau region, leading to a slightly higher cutoff value.

\section{B. Atomic SFA}

In the previous section, we explained the need for an atomic reference in order to remove the quadratic harmonic phase and collect the molecular specific two-center signature. This stands also in the case of molecular SFA computations, hence for completeness we recall in this section the basic equations of atomic SFA [60]. In SFA computations, an abrupt start of the laser field does not affect the spectrum, thus we choose a laser field without an envelope:

$$
\mathbf{E}(t)=E(t) \mathbf{e}_{x}=E_{\mathrm{L}} \cos (\omega t) \mathbf{e}_{x} .
$$

The corresponding vector potential $\mathbf{A}(t)$ is defined such that

$$
\mathbf{E}(t)=-\frac{\partial \mathbf{A}}{\partial t} .
$$

In the length gauge, the dipole reads

$$
\begin{aligned}
D_{\text {at }}(\Omega)= & \int d t \int_{0}^{t} d t^{\prime} \int d^{3} \mathbf{p} d_{\mathrm{rec}}[\mathbf{p}+\mathbf{A}(t)] \\
& \times d_{\text {ion }}\left(\mathbf{p}+\mathbf{A}\left(t^{\prime}\right), t^{\prime}\right) \mathrm{e}^{-i S\left(\mathbf{p}, t, t^{\prime}, \Omega\right)}
\end{aligned}
$$

Here $\Omega$ is the harmonic frequency, $S$ is the action,

$$
S\left(\mathbf{p}, t, t^{\prime}, \Omega\right)=\int_{t^{\prime}}^{t} d \tau\left(\frac{[\mathbf{p}+\mathbf{A}(\tau)]^{2}}{2}+I_{\mathrm{p}}\right)-\Omega t,
$$

and $d_{\text {ion }}$ and $d_{\text {rec }}$ are the projections of, respectively, the ionization and the recombination dipole matrix elements along the laser polarization, given by

$$
\begin{aligned}
& d_{\text {ion }}(\mathbf{k})=\left\langle\mathbf{k}\left|x E\left(t^{\prime}\right)\right| \varphi_{0}\right\rangle, \\
& d_{\text {rec }}(\mathbf{k})=\left\langle\varphi_{0}|-i \nabla| \mathbf{k}\right\rangle \cdot \mathbf{e}_{x},
\end{aligned}
$$

with $\left|\varphi_{0}\right\rangle$ and $|\mathbf{k}\rangle$ the atomic ground state and continuum wave functions, respectively.

The term $\exp \left[-i S\left(\mathbf{p}, t, t^{\prime}, \Omega\right)\right]$ is rapidly oscillating. Thus, the fivefold integration in Eq. (7) can be reduced to the evaluation of the integrand at the stationary solutions of $S$, for which

$$
\frac{\partial S}{\partial \mathbf{p}}=\mathbf{0}, \frac{\partial S}{\partial t}=0, \text { and } \frac{\partial S}{\partial t^{\prime}}=0 .
$$

This saddle-point approximation leads to the search of solutions of the following equations:

$$
\begin{aligned}
\int_{t^{\prime}}^{t} d \tau[\mathbf{p}+\mathbf{A}(\tau)] & =\mathbf{0}, \\
\frac{[\mathbf{p}+\mathbf{A}(t)]^{2}}{2}+I_{\mathrm{p}}-\Omega & =0, \\
\frac{\left[\mathbf{p}+\mathbf{A}\left(t^{\prime}\right)\right]^{2}}{2}+I_{\mathrm{p}} & =0 .
\end{aligned}
$$

We will denote by $\left(\mathbf{p}_{\mathrm{at}}, t_{\mathrm{at}}, t_{\mathrm{at}}^{\prime}\right)$ the solutions of Eqs. (11a)(11c) to emphasize that they are related to atomic trajectories. Note that the stationary momentum can be written as

$$
\mathbf{p}_{\mathrm{at}}\left(t_{\mathrm{at}}, t_{\mathrm{at}}^{\prime}\right)=-\frac{\int_{t_{\mathrm{at}}^{\prime}}^{t_{\mathrm{at}}} \mathbf{A}(\tau) d \tau}{t_{\mathrm{at}}-t_{\mathrm{at}}^{\prime}} .
$$

We also define $S_{\text {at }}=S\left(\mathbf{p}_{\mathrm{at}}, t_{\mathrm{at}}, t_{\mathrm{at}}^{\prime}, \Omega\right)$.

The total dipole [Eq. (7)] reduces to

$$
\begin{aligned}
D_{\mathrm{at}}(\Omega)= & C\left(t_{\mathrm{at}}, t_{\mathrm{at}}^{\prime}\right) d_{\mathrm{rec}}\left(\mathbf{p}_{\mathrm{at}}+\mathbf{A}\left(t_{\mathrm{at}}\right)\right) \\
& \times d_{\text {ion }}\left(\mathbf{p}_{\mathrm{at}}+\mathbf{A}\left(t_{\mathrm{at}}^{\prime}\right), t_{\mathrm{at}}^{\prime}\right) e^{-i S_{\mathrm{at}}} .
\end{aligned}
$$

For the sake of conciseness, no distinction is made between the short or the long trajectories in our notations. As a consequence, the sum over the short and long trajectories in the expression of $D_{\text {at }}$ will not be explicitly indicated in the following. Moreover, and again for clarity, we dropped the dependence of $t_{\mathrm{at}}$ and $t_{\mathrm{at}}^{\prime}$ (and consequently of $\mathbf{p}_{\mathrm{at}}$ ) on $\Omega$. In 
Eq. (13), $C$ is the saddle-point prefactor, which reads [79]

$$
C\left(t_{\mathrm{at}}, t_{\mathrm{at}}^{\prime}\right)=\left(\frac{2 \pi}{\epsilon+i\left(t_{\mathrm{at}}-t_{\mathrm{at}}^{\prime}\right)}\right)^{3 / 2} \frac{1}{\sqrt{\left.\operatorname{det}\left(S_{p}^{\prime \prime}\right)\right|_{\mathrm{at}}}},
$$

where $S_{p}^{\prime \prime}$ is the Hessian matrix of $S_{p}\left(t, t^{\prime}\right)=$ $S\left(\mathbf{p}_{\mathrm{at}}\left(t_{\mathrm{at}}, t_{\mathrm{at}}^{\prime}\right), t, t^{\prime}\right)$ :

$$
\left.\operatorname{det}\left(S_{p}^{\prime \prime}\right)\right|_{\text {at }}=\left.\left.\frac{\partial^{2} S_{p}}{\partial t^{2}}\right|_{\text {at }} \frac{\partial^{2} S_{p}}{\partial t^{\prime 2}}\right|_{\text {at }}-\left(\left.\frac{\partial^{2} S_{p}}{\partial t \partial t^{\prime}}\right|_{\text {at }}\right)^{2},
$$

with $\mathbf{p}_{\mathrm{at}}\left(t_{\mathrm{at}}, t_{\mathrm{at}}^{\prime}\right)$ given in Eq. (12). In the prefactor expression given in Eq. (14), the first factor arises from the evaluation of the integral, Eq. (7), over momentum at $\mathbf{p}_{\text {at }}$ and accounts for the spreading of the electronic wave packet in the continuum. The second factor comes from the evaluation of the integral over $t$ and $t^{\prime}$.

\section{Molecular SFA}

In this section, we recall the equations of molecular SFA as they have been developed in Refs. [70,71]. We will use the expression of the recombination and ionization dipole matrix elements as derived in Ref. [71]. These equations are used in the next section (Sec. II D) to obtain analytical expressions by means of Taylor expansions, which is the key point of our work.

The electronic ground state of the target molecule is approximated by a linear combination of atomic orbitals (LCAO), restricted to two atomic orbitals $\phi_{s}$ centered on the nuclei. Therefore, the recombination and ionization dipole matrix elements along $\mathbf{e}_{x}$ for a symmetric state $\left(\sigma_{g}\right)$ are expressed as [71]

$$
\begin{aligned}
d_{\text {rec }}(\mathbf{p}) & =\mathcal{R}(\mathbf{p})\left(e^{i \mathbf{p} \cdot \frac{\mathbf{R}}{2}}+e^{-i \mathbf{p} \cdot \frac{\mathbf{R}}{2}}\right), \\
d_{\text {ion }}\left(\mathbf{p}, t^{\prime}\right) & =\mathcal{I}_{1}\left(\mathbf{p}, t^{\prime}\right) e^{i \mathbf{p} \cdot \frac{\mathbf{R}}{2}}+\mathcal{I}_{2}\left(\mathbf{p}, t^{\prime}\right) e^{-i \mathbf{p} \cdot \frac{\mathbf{R}}{2}},
\end{aligned}
$$

where

$$
\begin{aligned}
\mathcal{R}(\mathbf{p}) & =\frac{\bar{\phi}_{s}(\mathbf{p})}{\sqrt{2[1+w(R)]}} \mathbf{p} \cdot \mathbf{e}_{x}, \\
\mathcal{I}_{\alpha}\left(\mathbf{p}, t^{\prime}\right) & =-\frac{\mathbf{E}\left(t^{\prime}\right)}{\sqrt{2[1+w(R)]}} \cdot\left[i \frac{\partial \bar{\phi}_{s}}{\partial \mathbf{p}}(\mathbf{p})+(-1)^{\alpha} \frac{\mathbf{R}}{2} \bar{\phi}_{s}(\mathbf{p})\right] .
\end{aligned}
$$

Here $\bar{\phi}_{s}$ is the momentum representation (Fourier transform) of the atomic orbital $\phi_{s}$, and $w(R)$ is the overlap between the two orbitals. In our computations, these orbitals are Gaussian functions [60]. The product of the transition dipole matrix elements thus gives

$$
\begin{aligned}
& d_{\mathrm{rec}}[\mathbf{p}+\mathbf{A}(t)] \times d_{\mathrm{ion}}\left(\mathbf{p}+\mathbf{A}\left(t^{\prime}\right), t^{\prime}\right) \\
& =\sum_{\alpha=1}^{2} \sum_{\beta=1}^{2} \mathcal{R}[\mathbf{p}+\mathbf{A}(t)] \times \mathcal{I}_{\alpha}\left(\mathbf{p}+\mathbf{A}\left(t^{\prime}\right), t^{\prime}\right) e^{-i \Phi_{\alpha \beta}\left(\mathbf{p}, t, t^{\prime}\right)},
\end{aligned}
$$

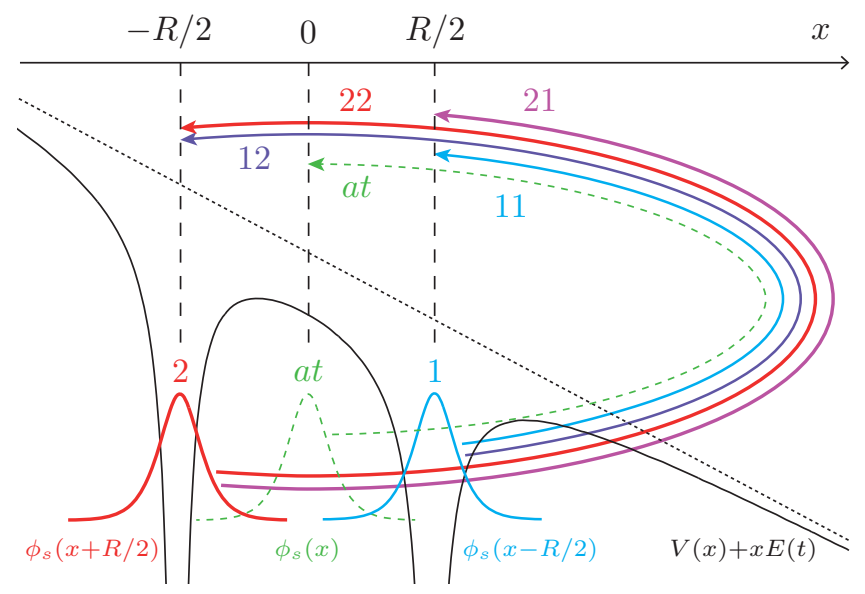

FIG. 1. One-dimensional sketch of the molecular potential and the four classes of trajectories $\alpha \rightarrow \beta$, with $\alpha, \beta \in\{1,2\}$ in molecular SFA. We present the case of aligned molecules along the laser polarization $\left(\mathbf{R}=R \mathbf{e}_{x}\right)$. We pictured the ionization toward the positive $x$-coordinate, i.e., with a negative laser field $E(t)$. We show by a black solid line the resulting potential, the sum of $x E(t)$ (dotted line) and of the double-well potential $V(x)$, and the atomic orbitals $\phi_{s}$ centered on each nucleus used for the computation of the transition dipole matrix elements.

where

$$
\begin{aligned}
\Phi_{\alpha \beta}\left(\mathbf{p}, t, t^{\prime}\right)= & (-1)^{\alpha}\left[\mathbf{p}+\mathbf{A}\left(t^{\prime}\right)\right] \cdot \frac{\mathbf{R}}{2} \\
& -(-1)^{\beta}[\mathbf{p}+\mathbf{A}(t)] \cdot \frac{\mathbf{R}}{2} .
\end{aligned}
$$

Here $\alpha$ and $\beta$ label the initial and final atomic center in the electron excursion, respectively. Thus, the total molecular dipole reads

$$
\begin{aligned}
D(\Omega)= & \sum_{\alpha=1}^{2} \sum_{\beta=1}^{2} \int d t \int_{0}^{t} d t^{\prime} \int d \mathbf{p} \mathcal{R}[\mathbf{p}+\mathbf{A}(t)] \\
& \times \mathcal{I}_{\alpha}\left(\mathbf{p}+\mathbf{A}\left(t^{\prime}\right), t^{\prime}\right) e^{-i S_{\alpha \beta}\left(\mathbf{p}, t, t^{\prime}, \Omega\right)} .
\end{aligned}
$$

It is the sum of four terms that can be evaluated independently by searching the stationary points of the modified actions:

$$
S_{\alpha \beta}\left(\mathbf{p}, t, t^{\prime}, \Omega\right)=S\left(\mathbf{p}, t, t^{\prime}, \Omega\right)+\Phi_{\alpha \beta}\left(\mathbf{p}, t, t^{\prime}\right) .
$$

This leads to the following saddle-point equations [71]:

$$
\begin{aligned}
\int_{t^{\prime}}^{t} d \tau[\mathbf{p}+\mathbf{A}(\tau)]+(-1)^{\alpha} \frac{\mathbf{R}}{2}-(-1)^{\beta} \frac{\mathbf{R}}{2} & =\mathbf{0}, \\
\frac{[\mathbf{p}+\mathbf{A}(t)]^{2}}{2}+I_{\mathrm{p}}-\Omega+(-1)^{\beta} \mathbf{E}(t) \cdot \frac{\mathbf{R}}{2} & =0, \\
\frac{\left[\mathbf{p}+\mathbf{A}\left(t^{\prime}\right)\right]^{2}}{2}+I_{\mathrm{p}}+(-1)^{\alpha} \mathbf{E}\left(t^{\prime}\right) \cdot \frac{\mathbf{R}}{2} & =0,
\end{aligned}
$$

for which we find four new groups of stationary solutions $\left(\mathbf{p}_{\alpha \beta}, t_{\alpha \beta}, t_{\alpha \beta}^{\prime}\right)$. They correspond to four classes of trajectories, represented in Fig. 1. They express the ionization from one center and the recombination to either the same center or the other one. Each class contains a short and a long path. Within the saddle-point approximation, the total dipole thus 
reduces to

$$
\begin{aligned}
D(\Omega)= & \sum_{\alpha=1}^{2} \sum_{\beta=1}^{2} C_{\alpha \beta}\left(t_{\alpha \beta}, t_{\alpha \beta}^{\prime}\right) \mathcal{R}\left[\mathbf{p}_{\alpha \beta}+\mathbf{A}\left(t_{\alpha \beta}\right)\right] \\
& \times \mathcal{I}_{\alpha}\left(\mathbf{p}_{\alpha \beta}+\mathbf{A}\left(t_{\alpha \beta}^{\prime}\right), t_{\alpha \beta}^{\prime}\right) e^{-i S_{\alpha \beta}\left(\mathbf{p}_{\alpha \beta}, t_{\alpha \beta}, t_{\alpha \beta}^{\prime}, \Omega\right)},
\end{aligned}
$$

with $C_{\alpha \beta}\left(t_{\alpha \beta}, t_{\alpha \beta}^{\prime}\right)$ the resulting saddle-point prefactors; see Eq. (14).

We will show in Sec. III that the observations on the phase jumps in the ab initio computations are well reproduced within the molecular SFA. This motivated us to further analyze the molecular SFA analytically and get a proper understanding of these behaviors. In the following, we express the deviations of the molecular solutions regarding the reference atomic ones by means of Taylor expansions.

\section{Taylor expansion of the molecular SFA}

\section{Stationary points}

As we explained, in Sec. II A we will calibrate our numerical results with the atomic ones in order to remove the quadratic harmonic phase. Moreover, since internuclear distances (a few $\AA$ ) are small compared to the travel path of the freed electrons in the continuum (several tens of $\AA$ ), the times for molecular trajectories are expected to remain close to those of an atomic trajectory. These considerations led us to perform Taylor expansions in powers of the internuclear distance $R$ of the molecular SFA equations, Eq. (24). To remain consistent with our TDSE simulations, where we restricted the study to a $1 \mathrm{D}$ case, we will consider in the following the specific case of a model molecule, with only one degree of freedom, aligned along the laser polarization, i.e., $\mathbf{R}=R \mathbf{e}_{x}$ and $\mathbf{p}=p \mathbf{e}_{x}$. Moreover, the "natural" absence of a perpendicular component of the momentum allows us to derive equations that reveal straightforward physical insights.

The solutions of the molecular saddle point equations (24) are expanded as

$$
\begin{aligned}
p_{\alpha \beta} & =p_{\mathrm{at}}+\Delta p_{\alpha \beta}^{(1)}+\Delta p_{\alpha \beta}^{(2)}+O\left(R^{3}\right), \\
t_{\alpha \beta} & =t_{\mathrm{at}}+\Delta t_{\alpha \beta}^{(1)}+\Delta t_{\alpha \beta}^{(2)}+O\left(R^{3}\right), \\
t_{\alpha \beta}^{\prime} & =t_{\mathrm{at}}^{\prime}+\Delta t_{\alpha \beta}^{(1)}+\Delta t_{\alpha \beta}^{\prime(2)}+O\left(R^{3}\right),
\end{aligned}
$$

where $\Delta p_{\alpha \beta}^{(1)}, \Delta t_{\alpha \beta}^{(1)}$, and $\Delta t_{\alpha \beta}^{\prime(1)}$ are proportional to $R$, and $\Delta p_{\alpha \beta}^{(2)}, \Delta t_{\alpha \beta}^{(2)}$, and $\Delta t_{\alpha \beta}^{\prime(2)}$ are proportional to $R^{2}$. Inserting this in Eq. (24), we obtain a linear set of equations for the first-order terms:

$$
\begin{aligned}
0= & {\left[p_{\mathrm{at}}+A\left(t_{\mathrm{at}}\right)\right] \Delta t_{\alpha \beta}^{(1)}-\left[p_{\mathrm{at}}+A\left(t_{\mathrm{at}}^{\prime}\right)\right] \Delta t_{\alpha \beta}^{\prime(1)} } \\
& +\left(t_{\mathrm{at}}-t_{\mathrm{at}}^{\prime}\right) \Delta p_{\alpha \beta}^{(1)}+\frac{(-1)^{\alpha}-(-1)^{\beta}}{2} R \\
0= & -\left[p_{\mathrm{at}}+A\left(t_{\mathrm{at}}\right)\right] E\left(t_{\mathrm{at}}\right) \Delta t_{\alpha \beta}^{(1)}+\left[p_{\mathrm{at}}+A\left(t_{\mathrm{at}}\right)\right] \Delta p_{\alpha \beta}^{(1)} \\
& +\frac{(-1)^{\beta}}{2} E\left(t_{\mathrm{at}}\right) R \\
0= & -\left[p_{\mathrm{at}}+A\left(t_{\mathrm{at}}^{\prime}\right)\right] E\left(t_{\mathrm{at}}^{\prime}\right) \Delta t_{\alpha \beta}^{(1)}+\left[p_{\mathrm{at}}+A\left(t_{\mathrm{at}}^{\prime}\right)\right] \Delta p_{\alpha \beta}^{(1)} \\
& +\frac{(-1)^{\alpha}}{2} E\left(t_{\mathrm{at}}^{\prime}\right) R .
\end{aligned}
$$

Resolving this set of equations (27) gives

$$
\begin{aligned}
\Delta p_{\alpha \beta}^{(1)} & =0, \\
\Delta t_{\alpha \beta}^{(1)} & =\frac{(-1)^{\beta} R}{2\left[p_{\mathrm{at}}+A\left(t_{\mathrm{at}}\right)\right]}, \\
\Delta t_{\alpha \beta}^{\prime(1)} & =\frac{(-1)^{\alpha} R}{2\left[p_{\mathrm{at}}+A\left(t_{\mathrm{at}}^{\prime}\right)\right]} .
\end{aligned}
$$

Using Eqs. (11), the last two equations can be rewritten as ${ }^{1}$

$$
\begin{aligned}
\Delta t_{\alpha \beta}^{(1)} & =\frac{(-1)^{\beta} R / 2}{\sqrt{2\left(\Omega-I_{p}\right)}}, \\
\Delta t_{\alpha \beta}^{(1)} & =i \frac{(-1)^{\alpha} R / 2}{\sqrt{2 I_{p}}} .
\end{aligned}
$$

The same procedure can be performed for the second-order terms; see Appendix A.

Tables I and II display the numerical values of the stationary momentum $p_{\alpha \beta}$ and of the ionization and recollision time deviations $t_{\alpha \beta}-t_{\mathrm{at}}$ and $t_{\alpha \beta}^{\prime}-t_{\mathrm{at}}^{\prime}$ and compare them with the values from the developments including either the first-order term only or both the first- and the second-order terms. An excellent agreement is found between the numerically exact results and the development including up to the second order. Yet, we note that the first-order term is in very good agreement with the exact values, with errors limited to a few percent (around 0.02 a.u.). We will therefore restrict our development to this order. As will be shown, the first-order treatment has the valuable advantage of providing a direct and simple physical picture of the underlying timings of ionization and recollision in diatomic molecules.

First, the ionization time is shifted by a pure imaginary delay $\Delta t_{\alpha \beta}^{\prime(1)}$, as seen in Eq. (29b), which can be interpreted as a correction to the tunneling time with respect to the atomic case. If the electron is ionized from the center $\alpha=1$, located at $R / 2$, we find that $\operatorname{Im}\left(\Delta t_{1 \beta}^{\prime(1)}\right)<0$, which means that the time spent "below the barrier" is smaller. This is consistent with a smaller barrier to cross, as seen in Fig. 1. The opposite situation is observed for an electron ionized from center $\alpha=2$ for which $\operatorname{Im}\left(\Delta t_{2 \beta}^{\prime(1)}\right)>0$, where a larger barrier is to be crossed.

Second, the shift for the recollision times with respect to the atomic times, $\Delta t_{\alpha \beta}^{(1)}$, Eq. (29a), is now real. For an electron recombining with the center located at $R / 2$ again, i.e., $\beta=1$, we find that $\Delta t_{\alpha 1}^{(1)}<0$, which means that the electron recollides earlier compared to the atomic case. Indeed, as pictured in Fig. 1, the electron travels a shorter path. On the contrary, an electron recolliding with center $\beta=2$ for which $\Delta t_{\alpha 2}^{(1)}>0$ travels a longer path.

Note that for both time delays $\Delta t_{\alpha \beta}^{(1)}$ and $\Delta t_{\alpha \beta}^{\prime(1)}$, their formal expressions, Eqs. (28), can be regarded as the ratio between the additional distance to cross in the molecule, $\pm R / 2$, and the

\footnotetext{
${ }^{1}$ In these equations and in their interpretation below, we used the sign convention illustrated in Fig. 1, where the barrier is lowered for $x>0$, i.e., when the electric field is negative at ionization time.
} 
TABLE I. Numerical values of the stationary momenta $p_{\alpha \beta}$ solution of the saddle point Eqs. (24), compared to its development Eq. (26), restricted either to first or second order, for six harmonics (column denoted by the $\mathrm{H}$ symbol) within the plateau of the spectrum generated at a laser intensity of $5 \times 10^{14} \mathrm{~W} \mathrm{~cm}^{-2}$.

\begin{tabular}{|c|c|c|c|c|c|}
\hline & \multirow[b]{2}{*}{$\mathrm{H}$} & \multicolumn{2}{|c|}{ Short trajectories } & \multicolumn{2}{|c|}{ Long trajectories } \\
\hline & & $\mathrm{Re}$ & $\operatorname{Im}$ & $\operatorname{Re}$ & $\operatorname{Im}$ \\
\hline \multirow[t]{6}{*}{$p_{11}$} & 21 & 1.8976 & 0.14767 & 0.091138 & $5.0125 \times 10^{-4}$ \\
\hline & 31 & 1.6986 & 0.089790 & 0.14357 & $7.7589 \times 10^{-4}$ \\
\hline & 41 & 1.5214 & 0.061121 & 0.20458 & $1.0670 \times 10^{-3}$ \\
\hline & 51 & 1.3508 & 0.044192 & 0.27847 & $1.3447 \times 10^{-3}$ \\
\hline & 61 & 1.1706 & 0.033242 & 0.37518 & $1.4493 \times 10^{-3}$ \\
\hline & 71 & 0.93811 & 0.027673 & 0.53362 & $-5.5265 \times 10^{-4}$ \\
\hline \multirow[t]{6}{*}{$p_{12}$} & 21 & 1.8976 & 0.14769 & 0.091138 & $5.0125 \times 10^{-4}$ \\
\hline & 31 & 1.6986 & 0.089793 & 0.14357 & $7.7590 \times 10^{-4}$ \\
\hline & 41 & 1.5214 & 0.061122 & 0.20458 & $1.0670 \times 10^{-3}$ \\
\hline & 51 & 1.3508 & 0.044192 & 0.27847 & $1.3447 \times 10^{-3}$ \\
\hline & 61 & 1.1706 & 0.033242 & 0.37518 & $1.4493 \times 10^{-3}$ \\
\hline & 71 & 0.93811 & 0.027673 & 0.53362 & $-5.5264 \times 10^{-4}$ \\
\hline \multirow[t]{6}{*}{$p_{21}$} & 21 & 1.8975 & 0.14772 & 0.091138 & $5.0176 \times 10^{-4}$ \\
\hline & 31 & 1.6986 & 0.089830 & 0.14357 & $7.7668 \times 10^{-4}$ \\
\hline & 41 & 1.5214 & 0.061154 & 0.20458 & $1.0681 \times 10^{-3}$ \\
\hline & 51 & 1.3508 & 0.044219 & 0.27847 & $1.3460 \times 10^{-3}$ \\
\hline & 61 & 1.1706 & 0.033266 & 0.37518 & $1.4507 \times 10^{-3}$ \\
\hline & 71 & 0.93811 & 0.027695 & 0.53362 & $-5.5317 \times 10^{-4}$ \\
\hline \multirow[t]{6}{*}{$p_{22}$} & 21 & 1.8976 & 0.14774 & 0.091138 & $5.0176 \times 10^{-4}$ \\
\hline & 31 & 1.6986 & 0.089833 & 0.14357 & $7.7668 \times 10^{-4}$ \\
\hline & 41 & 1.5214 & 0.061155 & 0.20458 & $1.0681 \times 10^{-3}$ \\
\hline & 51 & 1.3508 & 0.044220 & 0.27847 & $1.3460 \times 10^{-3}$ \\
\hline & 61 & 1.1706 & 0.033266 & 0.37518 & $1.4507 \times 10^{-3}$ \\
\hline & 71 & 0.93811 & 0.027695 & 0.53362 & $-5.5316 \times 10^{-4}$ \\
\hline \multirow[t]{6}{*}{$p_{2^{\text {nd }}}$} & 21 & 1.8976 & 0.14770 & 0.091138 & $5.0152 \times 10^{-4}$ \\
\hline & 31 & 1.6986 & 0.089812 & 0.14357 & $7.7631 \times 10^{-4}$ \\
\hline & 41 & 1.5214 & 0.061138 & 0.20458 & $1.0676 \times 10^{-3}$ \\
\hline & 51 & 1.3508 & 0.044206 & 0.27847 & $1.3454 \times 10^{-3}$ \\
\hline & 61 & 1.1706 & 0.033255 & 0.37518 & $1.4501 \times 10^{-3}$ \\
\hline & 71 & 0.93811 & 0.027685 & 0.53362 & $-5.5280 \times 10^{-4}$ \\
\hline$p_{\text {at }}$ & 21 & 1.8972 & 0.14788 & 0.091148 & $5.0516 \times 10^{-4}$ \\
\hline \multirow[t]{5}{*}{$=p_{1^{\mathrm{st}}}$} & 31 & 1.6982 & 0.089978 & 0.14359 & $7.8195 \times 10^{-4}$ \\
\hline & 41 & 1.5210 & 0.061302 & 0.20461 & $1.0753 \times 10^{-3}$ \\
\hline & 51 & 1.3504 & 0.044360 & 0.27853 & $1.3549 \times 10^{-3}$ \\
\hline & 61 & 1.1702 & 0.033397 & 0.37528 & $1.4596 \times 10^{-3}$ \\
\hline & 71 & 0.93754 & 0.027841 & 0.53390 & $-5.6899 \times 10^{-4}$ \\
\hline
\end{tabular}

velocity of the electron given in Eqs. (11b) and (11c), either classically allowed or forbidden.

We now turn to the Taylor expansion of the different terms entering the expression of the total dipole in Eq. (25) in order to find out how these differences of timing affect the phase of HHG.

\section{Total dipole}

We first expand the modified action $S_{\alpha \beta}$ to first order in $R$ :

$$
\begin{aligned}
S_{\alpha \beta}\left(p_{\alpha \beta}, t_{\alpha \beta}, t_{\alpha \beta}^{\prime}, \Omega\right)= & S_{\text {at }}+\Phi_{\alpha \beta}\left(p_{\text {at }}, t_{\mathrm{at}}, t_{\mathrm{at}}^{\prime}\right) \\
& +\left.\frac{\partial S}{\partial t}\right|_{\text {at }} \Delta t_{\alpha \beta}^{(1)}+\left.\frac{\partial S}{\partial t^{\prime}}\right|_{\mathrm{at}} \Delta t_{\alpha \beta}^{(1)}+O\left(R^{2}\right) .
\end{aligned}
$$

The partial derivatives of the action $S$ with respect to $t$ and $t^{\prime}$ cancel at the atomic stationary points (10), so we simply get

$$
S_{\alpha \beta}\left(p_{\alpha \beta}, t_{\alpha \beta}, t_{\alpha \beta}^{\prime}, \Omega\right)=S_{\text {at }}+\Phi_{\alpha \beta}\left(p_{\mathrm{at}}, t_{\mathrm{at}}, t_{\mathrm{at}}^{\prime}\right)+O\left(R^{2}\right) .
$$

Then we expand the ionization and recombination dipole matrix elements [Eqs. (18) and (19)]:

$$
\begin{aligned}
\mathcal{R}\left[p_{\alpha \beta}+A\left(t_{\alpha \beta}\right)\right]= & \mathcal{R}_{\mathrm{at}}\left[p_{\mathrm{at}}+A\left(t_{\mathrm{at}}\right)\right] \\
& +(-1)^{\beta} \mathcal{R}^{(1)}\left(p_{\mathrm{at}}+A\left(t_{\mathrm{at}}\right), t_{\mathrm{at}}\right) R \\
& +O\left(R^{2}\right),
\end{aligned}
$$

where

$$
\begin{aligned}
\mathcal{R}_{\mathrm{at}}(p) & =\frac{p \bar{\phi}_{s}(p)}{2}, \\
\mathcal{R}^{(1)}(p, t) & =-\frac{E(t)}{4}\left(\frac{\bar{\phi}_{s}(p)}{p}+\frac{\partial \bar{\phi}_{s}}{\partial p}(p)\right),
\end{aligned}
$$

and

$$
\begin{gathered}
\mathcal{I}_{\alpha}\left(p_{\alpha \beta}+A\left(t_{\alpha \beta}^{\prime}\right), t_{\alpha \beta}^{\prime}\right)=\mathcal{I}_{\mathrm{at}}\left(p_{\mathrm{at}}+A\left(t_{\mathrm{at}}^{\prime}\right), t_{\mathrm{at}}^{\prime}\right) \\
-(-1)^{\alpha} \mathcal{I}^{(1)}\left(p_{\mathrm{at}}+A\left(t_{\mathrm{at}}^{\prime}\right), t_{\mathrm{at}}^{\prime}\right) R+O\left(R^{2}\right),
\end{gathered}
$$

where

$$
\begin{aligned}
\mathcal{I}_{\text {at }}\left(p, t^{\prime}\right)= & \frac{-i E\left(t^{\prime}\right)}{2} \frac{\partial \bar{\phi}_{s}}{\partial p}(p), \\
\mathcal{I}^{(1)}\left(p, t^{\prime}\right)= & \frac{E\left(t^{\prime}\right)}{4} \bar{\phi}_{s}(p)+\frac{i \omega^{2} A\left(t^{\prime}\right)}{4 p} \frac{\partial \bar{\phi}_{s}}{\partial p}(p) \\
& -i \frac{E^{2}\left(t^{\prime}\right)}{4 p} \frac{\partial^{2} \bar{\phi}_{s}}{\partial p^{2}}(p) .
\end{aligned}
$$

We checked numerically that the molecular prefactor $C_{\alpha \beta}\left(t_{\alpha \beta}, t_{\alpha \beta}^{\prime}\right)$ is actually very close to the atomic one. We will thus, as a first approximation, consider it to be equal to $C\left(t_{\mathrm{at}}, t_{\mathrm{at}}^{\prime}\right)(14)$. The exact effect of this approximation will be discussed in Sec. IV B.

Finally, after regrouping all the terms, the first-order approximation of the total dipole (25) can be refolded into

$$
\begin{aligned}
\widetilde{D}(\Omega)= & C\left(t_{\mathrm{at}}, t_{\mathrm{at}}^{\prime}\right) \widetilde{d}_{\mathrm{rec}}\left(p_{\mathrm{at}}+A\left(t_{\mathrm{at}}\right), t_{\mathrm{at}}\right) \\
& \times \widetilde{d}_{\mathrm{ion}}\left(p_{\mathrm{at}}+A\left(t_{\mathrm{at}}^{\prime}\right), t_{\mathrm{at}}^{\prime}\right) e^{-i S_{\mathrm{at}} .}
\end{aligned}
$$

We have thus retrieved an expression that is similar to the atomic dipole given by Eq. (7). It is evaluated at atomic stationary points $\left(p_{\mathrm{at}}, t_{\mathrm{at}}, t_{\mathrm{at}}^{\prime}\right)$ and involves the atomic action $S_{\text {at. }}$ However, it involves modified transition dipole matrix elements $\widetilde{d}_{\text {ion }}$ and $\widetilde{d}_{\text {rec }}$ expressed as

$$
\tilde{d}_{\mathrm{rec}}(p, t)=2 \mathcal{R}(p) \cos \left(\frac{p R}{2}\right)+2 i \mathcal{R}^{(1)}(p, t) R \sin \left(\frac{p R}{2}\right),
$$

$$
\begin{aligned}
\tilde{d}_{\text {ion }}\left(p, t^{\prime}\right)= & 2 \mathcal{I}_{\text {at }}\left(p, t^{\prime}\right) \cos \left(\frac{p R}{2}\right) \\
& +2 i \mathcal{I}^{(1)}\left(p, t^{\prime}\right) R \sin \left(\frac{p R}{2}\right) .
\end{aligned}
$$


TABLE II. Numerical values of the time deviations $t_{\alpha \beta}-t_{\mathrm{at}}$ and $t_{\alpha \beta}^{\prime}-t_{\mathrm{at}}$ for the same harmonics and the same generation conditions as in Table I, compared with our development (26), to either the first or the second order. We present the numerical values for the short (S) and the long (L) trajectories. We do not present the imaginary part of $t_{\alpha \beta}-t_{\mathrm{at}}\left(<3 \times 10^{-3}\right.$ a.u.), nor the real part of $t_{\alpha \beta}^{\prime}-t_{\mathrm{at}}^{\prime}\left(<5 \times 10^{-3}\right.$ a.u. $)$. The deviations from the atomic case are small compared to the imaginary part of the ionization times (7.83-15.8 a.u.) and the real part of the recollision times (32.9-107 a.u.), respectively.

\begin{tabular}{|c|c|c|c|c|c|c|c|c|c|c|c|}
\hline \multirow[b]{3}{*}{$\alpha \beta$} & \multirow[b]{3}{*}{$\mathrm{H}$} & \multirow{2}{*}{\multicolumn{4}{|c|}{$\begin{array}{l}\operatorname{Re}\left(t_{\alpha \beta}-t_{\mathrm{at}}\right) \\
\quad \text { Second order }\end{array}$}} & \multirow{3}{*}{ First order } & \multicolumn{5}{|c|}{$\operatorname{Im}\left(t_{\alpha \beta}^{\prime}-t_{\mathrm{at}}^{\prime}\right)$} \\
\hline & & & & & & & \multicolumn{2}{|c|}{ Numerical } & \multicolumn{2}{|c|}{ Second order } & \multirow[t]{2}{*}{ First order } \\
\hline & & $S$ & $\mathrm{~L}$ & $\mathrm{~S}$ & $\mathrm{~L}$ & & $S$ & $\mathrm{~L}$ & $S$ & $\mathrm{~L}$ & \\
\hline \multirow[t]{6}{*}{11} & 21 & -0.643 & -0.601 & -0.639 & -0.599 & -0.624 & -0.669 & -0.681 & -0.668 & -0.679 & -0.657 \\
\hline & 31 & -0.463 & -0.441 & -0.463 & -0.441 & -0.452 & -0.670 & -0.681 & -0.670 & -0.679 & -0.657 \\
\hline & 41 & -0.381 & -0.365 & -0.381 & -0.365 & -0.372 & -0.672 & -0.681 & -0.672 & -0.679 & -0.657 \\
\hline & 51 & -0.331 & -0.317 & -0.331 & -0.317 & -0.324 & -0.674 & -0.681 & -0.673 & -0.679 & -0.657 \\
\hline & 61 & -0.298 & -0.284 & -0.298 & -0.284 & -0.290 & -0.676 & -0.681 & -0.675 & -0.679 & -0.657 \\
\hline & 71 & -0.277 & -0.255 & -0.277 & -0.255 & -0.265 & -0.678 & -0.680 & -0.677 & -0.680 & -0.657 \\
\hline \multirow[t]{6}{*}{12} & 21 & 0.605 & 0.650 & 0.608 & 0.648 & 0.624 & -0.668 & -0.681 & -0.668 & -0.679 & -0.657 \\
\hline & 31 & 0.441 & 0.463 & 0.441 & 0.463 & 0.452 & -0.670 & -0.681 & -0.670 & -0.679 & -0.657 \\
\hline & 41 & 0.364 & 0.380 & 0.363 & 0.379 & 0.372 & -0.672 & -0.681 & -0.672 & -0.679 & -0.657 \\
\hline & 51 & 0.316 & 0.330 & 0.316 & 0.330 & 0.324 & -0.674 & -0.681 & -0.673 & -0.679 & -0.657 \\
\hline & 61 & 0.282 & 0.297 & 0.282 & 0.297 & 0.290 & -0.676 & -0.681 & -0.675 & -0.679 & -0.657 \\
\hline & 71 & 0.254 & 0.276 & 0.253 & 0.276 & 0.265 & -0.678 & -0.680 & -0.677 & -0.680 & -0.657 \\
\hline \multirow[t]{6}{*}{21} & 21 & -0.642 & -0.601 & -0.639 & -0.599 & -0.624 & 0.647 & 0.637 & 0.646 & 0.636 & 0.657 \\
\hline & 31 & -0.463 & -0.441 & -0.463 & -0.441 & -0.452 & 0.646 & 0.637 & 0.645 & 0.636 & 0.657 \\
\hline & 41 & -0.381 & -0.365 & -0.381 & -0.365 & -0.372 & 0.644 & 0.637 & 0.643 & 0.636 & 0.657 \\
\hline & 51 & -0.331 & -0.317 & -0.331 & -0.317 & -0.324 & 0.642 & 0.637 & 0.641 & 0.636 & 0.657 \\
\hline & 61 & -0.298 & -0.284 & -0.298 & -0.284 & -0.290 & 0.641 & 0.637 & 0.640 & 0.636 & 0.657 \\
\hline & 71 & -0.277 & -0.255 & -0.277 & -0.255 & -0.265 & 0.639 & 0.638 & 0.638 & 0.636 & 0.657 \\
\hline \multirow[t]{6}{*}{22} & 21 & 0.605 & 0.650 & 0.608 & 0.648 & 0.624 & 0.647 & 0.637 & 0.646 & 0.636 & 0.657 \\
\hline & 31 & 0.441 & 0.463 & 0.441 & 0.463 & 0.452 & 0.646 & 0.637 & 0.645 & 0.636 & 0.657 \\
\hline & 41 & 0.364 & 0.380 & 0.363 & 0.379 & 0.372 & 0.644 & 0.637 & 0.643 & 0.636 & 0.657 \\
\hline & 51 & 0.316 & 0.330 & 0.316 & 0.330 & 0.324 & 0.642 & 0.637 & 0.641 & 0.636 & 0.657 \\
\hline & 61 & 0.283 & 0.297 & 0.282 & 0.297 & 0.290 & 0.641 & 0.637 & 0.640 & 0.636 & 0.657 \\
\hline & 71 & 0.254 & 0.276 & 0.253 & 0.276 & 0.265 & 0.639 & 0.638 & 0.638 & 0.636 & 0.657 \\
\hline
\end{tabular}

These expressions are reminiscent of the ones of [56,57], where field dressed states were used a priori to define the dipole.

\section{NUMERICAL SIMULATIONS}

\section{A. Ab initio computations}

As presented in Sec. II A, we first investigate the signature of two-center interference in both the short and long trajectory contributions to the HHG spectrum with our TDSE computations. The results are presented in Figs. 2 and 3. We report the intensity and phase of the short and long trajectory contributions to HHG spectra for several internuclear distances between 1.4 and 1.7 a.u., generated at a laser peak intensity of $2.65 \times 10^{14} \mathrm{~W} \mathrm{~cm}^{-2}$. The calibration with a reference atom, as explained in Sec. II, implies to divide the harmonic intensity by that of the atom. Consistently, the reported phase is the phase difference between the molecular and the atomic computations, which removes the quadratic contribution and allows the observation of the two-center interference effects.

In the intensity of each spectrum we observe a minimum, which is shallow in the case of the short trajectories [Fig. 2(a)] and much more pronounced for the long trajectories, especially for particular values of $R$ [Fig. 3(a)]. This minimum is the signature of the destructive interference induced by the two centers of the molecule in which we are interested. Its position has long been ascribed to the zeros of the recombination dipole matrix element $d_{\mathrm{rec}}$ given by Eq. (16), which relies on plane waves (PW) to describe the continuum and on the LCAO approximation for the ground state [65]. Within these approximations, the zeros of $d_{\text {rec }}$ occur at particular momentum values $p_{k}=(2 k+1) \pi / R, k \in \mathbb{Z}$. We focus on the first value $p_{0}$ as it is the only one contained in our harmonic spectra generated by a relatively short-wavelength laser like Ti:sapphire. The corresponding harmonic frequency is

$$
\Omega_{0}^{(\mathrm{PW})}=I_{\mathrm{p}}+\frac{p_{0}^{2}}{2}=I_{\mathrm{p}}+\frac{\pi^{2}}{2 R^{2}} .
$$

For the illustrative case $R=1.57$ a.u., this leads to a minimum at harmonic 45, while our TDSE simulations give a minimum at "harmonic" 38.5 .

A way to overcome this discrepancy is to claim that effective PW representing the returning electron must be energyshifted by $I_{\mathrm{p}}$ [65], leading to the approximate expression

$$
\bar{\Omega}_{0} \simeq \frac{\pi^{2}}{2 R^{2}} .
$$

Still, this correction is not sufficient as it predicts a minimum at harmonic 35 .

Therefore, we used numerical simulations to better understand why the analytical formula, either with or with- 


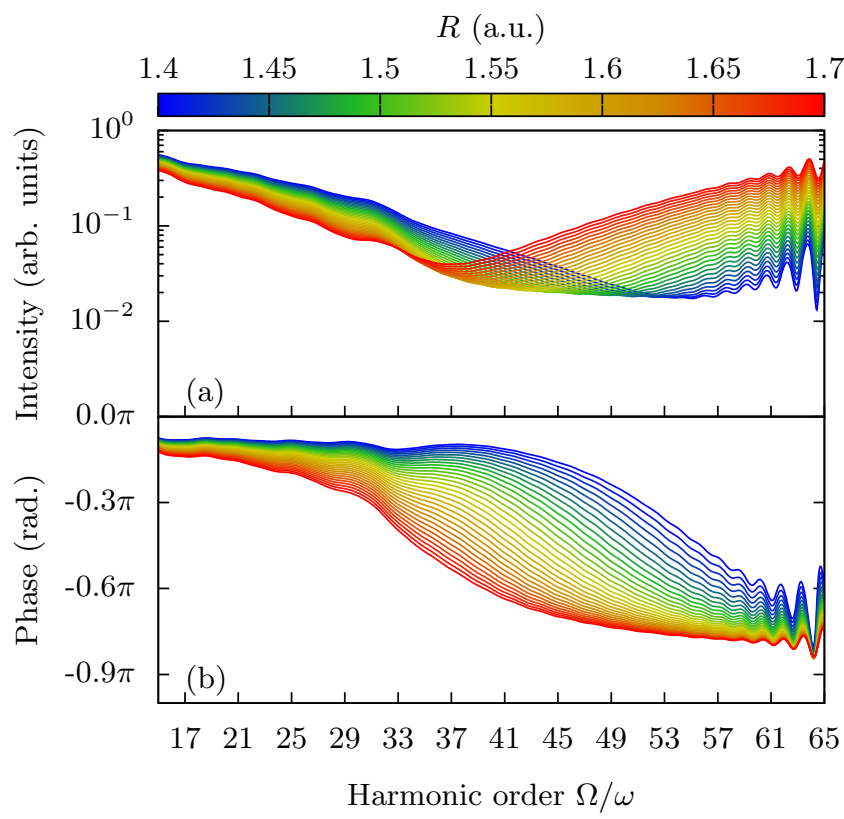

FIG. 2. Ab initio computations of the contribution of the short trajectories to high-order harmonic spectra for a two-cycle laser pulse of $2.65 \times 10^{14} \mathrm{~W} \mathrm{~cm}^{-2}$ peak intensity for a diatomic molecule with different internuclear distances $R$ from 1.4 to 1.7 a.u. We report the harmonic intensity (a) and phase (b) calibrated to an atomic reference.

out the $I_{\mathrm{p}}$ shift [Eqs. (42) and (41), respectively] does not reproduce the position of the minimum. We computed numerically the eigenstates of the field-free system by solving the time-independent Schrödinger equation. The ground state was computed by diagonalizing the field-free Hamiltonian, while the continuum states were computed with a fourth-order Runge-Kutta algorithm and normalized with the Strömgren procedure [80]. We then used these numerically exact states to compute the "exact" recombination dipole, and to extract its zero for different values of $R$. This is shown with a solid green line in Fig. 4. As predicted by the three-step model, the zeros of the exact recombination dipole perfectly match the position of the minima observed in the HHG spectra, as extracted from the long trajectory contributions. Note that, for small $R \lesssim 1.6$ a.u., the mismatch is related to the difficulty of finding the minimum in the TDSE HHG spectrum, because, as can be seen in Fig. 3, it is located too close to the cutoff. However, if we compute the recombination dipole using the "exact" continuum states and the LCAO ground state (dashed blue line), the position of the minimum is clearly underestimated, by at least 10 harmonic orders. On the contrary, if we compute the recombination dipole with the "exact" ground state and PW for the continuum (dotted pink and purple lines), the position of the minimum is now greatly overestimated, whether we use the $I_{\mathrm{p}}$ shift correction or not. Finally, when we perform both the LCAO and the PW approximations (dash-dotted orange and yellow lines), we see that the errors from both approximations compensate each other, providing a relatively good, but somehow accidental, agreement with the TDSE results. We note that this error compensation leads to a better agreement with the $I_{\mathrm{p}}$ shift correction. We thus want to

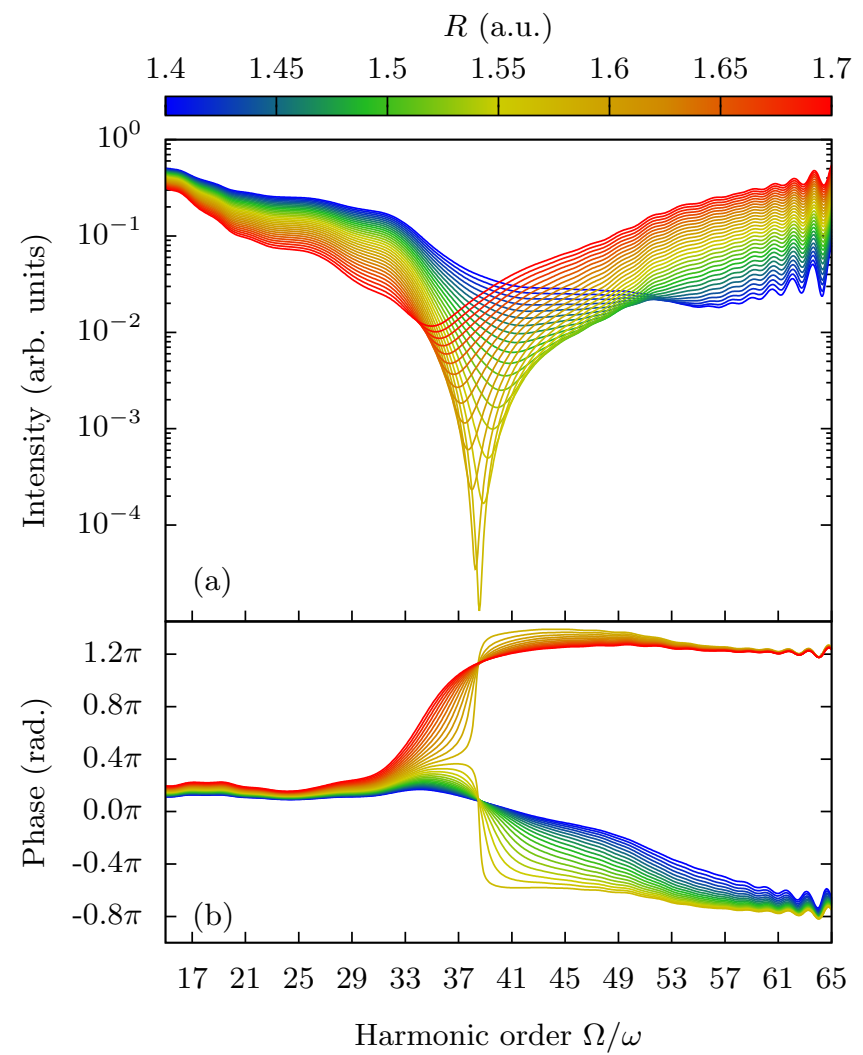

FIG. 3. Same as in Fig. 2 for the long trajectories.

draw attention to the fact that one always has to remain careful when considering a model with several approximations. These error compensations can actually happen more often than we think [81].

We now turn to the shape of the minimum and of the corresponding phase jumps. As already observed in $[64,65,69]$, the phase jumps are smoothed, covering several harmonics, with a magnitude lower than $\pi$ rad [Figs. 2(b) and 3(b)]. The interference condition exhibits more surprising features. While the phase jump is smoothed and negative for all values of $R$ for short trajectories [Fig. 2(b)], the situation is completely different for the long trajectories. It is smoothed and positive for small $R$, then it varies steeply at a critical internuclear distance $R_{c}=1.57$ a.u., beyond which the behavior reverses into a smooth negative jump as for the short trajectories [Fig. 3(b)]. Moreover, the depth of the minimum in the amplitude is directly related to the shape of the phase jump: the steeper the jump, the deeper the minimum.

In addition, we performed a time-frequency analysis of the harmonic dipole using Gabor transforms as in $[23,56,57]$ to retrieve, for different values of $R$, the emission times of the harmonics for which the destructive interference occurs. Figure 5 displays the Gabor transform of the dipole for the particular case of $R=R_{c}$. We found that the recollision time for the long trajectory associated with the interference frequency corresponds to an almost zero electric field $\left(E_{\text {rec }} \simeq\right.$ $9.5 \times 10^{-3}$ a.u.). Moreover, greater (lower) values of $R$ lead to recombination in the presence of a negative (positive) electric field for the long trajectories, while the field at recombination is always positive for the short trajectories. 


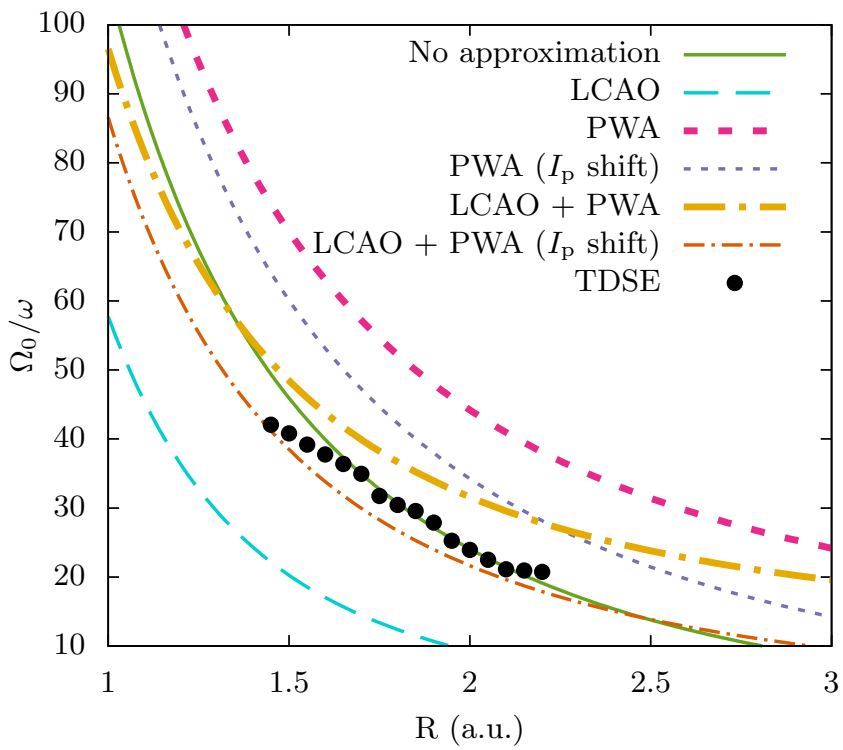

FIG. 4. Harmonic order of the minimum as a function of $R$. Black dots: minimum in the harmonic spectrum extracted from TDSE calculations, lines: zero of the recombination dipole matrix element computed at different levels of approximation.

To further examine the relation between the value of the electric field at the recombination and the shape of the phase jump, we tuned the laser peak intensity $I_{\mathrm{L}}$ from 2.5 to $4.5 \times 10^{14} \mathrm{~W} \mathrm{~cm}^{-2}$ at fixed $R$. We chose $R=R_{\mathrm{eq}}=1.4$ a.u. The corresponding trajectory-resolved phases are shown in Fig. 6. As expected, the position of the destructive interference

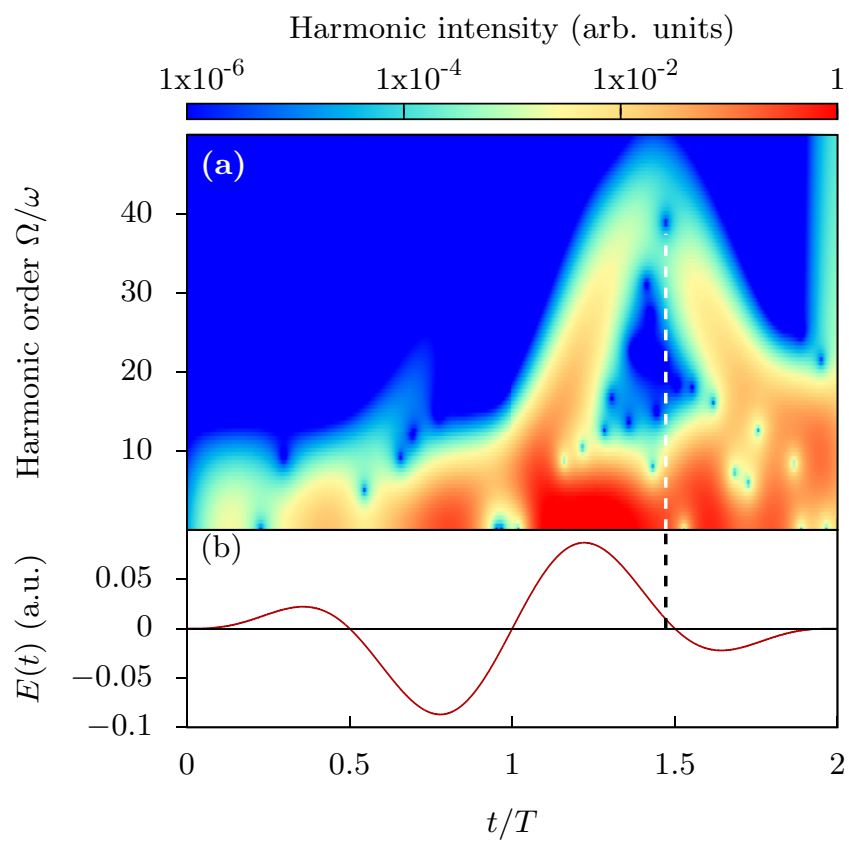

FIG. 5. Gabor transform of the $a b$ initio dipole for the critical internuclear distance $R_{c}=1.57$ a.u. (a) and instantaneous value of the electric (b). The peak intensity is $2.65 \times 10^{14} \mathrm{~W} \mathrm{~cm}^{-2}$. We marked with dashed lines the time associated with the minimum observed in the long trajectories.

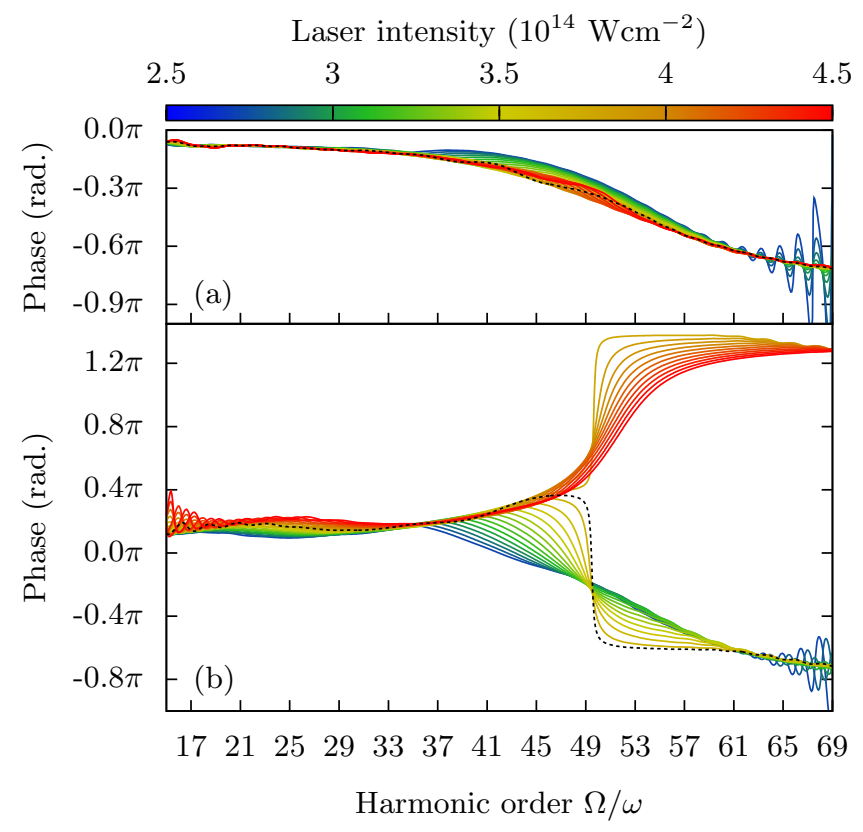

FIG. 6. Ab initio computations of the harmonic phase of a model $\mathrm{H}_{2}$ relative to the atomic reference for a two-cycle laser pulse with peak intensities in the range $(2.5-4.5) \times 10^{14} \mathrm{Wcm}^{-2}$ for short (a) and long trajectories (b). The dashed line corresponds to intensity $I_{c}=3.74 \times 10^{14} \mathrm{~W} \mathrm{~cm}^{-2}$.

does not change with $I_{\mathrm{L}}$ and we observe a behavior for the phase jumps that is similar to the one when varying $R$. It is smoothed and negative for all intensities at the short trajectories, while an equivalent smoothing and inversion behavior is present for the long trajectories. We found a critical intensity $I_{c}=3.74 \times 10^{14} \mathrm{~W} \mathrm{~cm}^{-2}$ at which the inversion occurs with a discontinuous $\pm \pi$ rad phase jump [see the black-dashed curve in Fig. 6(b)].

Using time-frequency analysis once again, this change of behavior is directly related to the change of sign of the instantaneous electric field at recombination time, which is almost zero at $\Omega_{0}$ for the intensity $I_{c}$. We find that, in this case, the corresponding electric field amounts to $E_{\mathrm{rec}}=7.4 \times 10^{-3}$ a.u. This corresponds to $\equiv I_{\mathrm{rec}}=$ $1.92 \times 10^{12} \mathrm{Wcm}^{-2}$, which is $\sim 0.5 \%$ of $I_{c}$.

\section{B. Molecular SFA}

To get more physical insights, the same study has been carried out within the molecular SFA derived in Sec. IIC, following [71]. We report in Fig. 7 the phase of the short and long trajectories as a function of the laser intensity. Again this phase is relative to the phase of atomic SFA presented in Sec. II B, following [60]. The separation of the short and long trajectory contributions in SFA is straightforward, being inherent to the search of saddle-point solutions. Consequently, no residual oscillation is encountered, in contrast to the TDSE results. Nevertheless, the SFA model prevents the computation of harmonics above the cutoff, which limits the observation of the entire phase jumps for the lowest intensities.

We observe that the behavior of the phase is very similar to that of the TDSE simulations. The major difference is the 


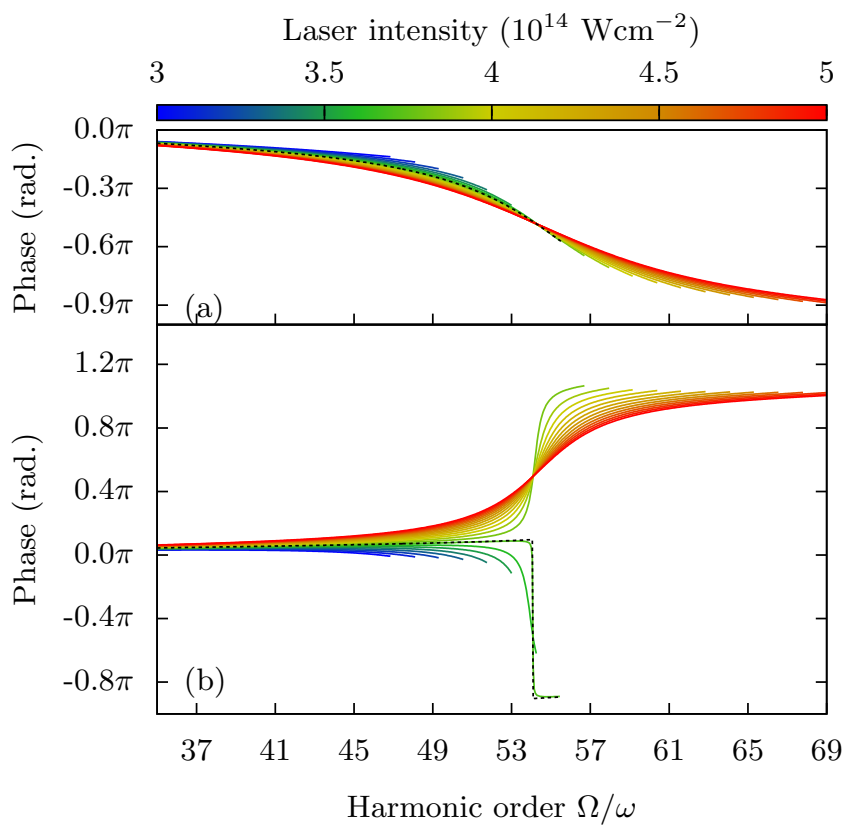

FIG. 7. Molecular SFA computations of the harmonic phase of a model $\mathrm{H}_{2}$ normalized by the atomic SFA for a two-cycle laser pulse with peak intensities in the range $(3-5) \times 10^{14} \mathrm{Wcm}^{-2}$ for short (a) and long trajectories (b). The dashed line corresponds to the intensity $I_{c}^{\prime}=3.704 \times 10^{14} \mathrm{~W} \mathrm{~cm}^{-2}$.

position of the destructive interference due to the LCAO and PW approximations in SFA, as mentioned before. Hence the destructive interference is observed exactly at the frequency given by Eq. (41). Furthermore, the intensity for which the phase is discontinuous and jumps by $\pm \pi$ rad is shifted to $I_{c}^{\prime}=3.704 \times 10^{14} \mathrm{~W} \mathrm{~cm}^{-2}$.

The SFA computations allowed us to obtain the precise value of the electric field at recombination time for the long trajectories and for the inversion intensity $I_{c}^{\prime}$. It is found to be $E_{\text {rec }}=0.0034$ a.u., which is comparable to the one observed in the ab initio computations. The discrepancies are attributed to the difference between $a b$ initio and SFA trajectory times [82].

Finally, we have performed simulations at the laser intensity for which the electric field is exactly zero at recombination time $-I_{z}=3.641 \times 10^{14} \mathrm{~W} \mathrm{~cm}^{-2}$, which is slightly lower than $I_{c}^{\prime}$, with a difference of only $6 \times 10^{12} \mathrm{~W} \mathrm{~cm}^{-2}$, i.e., $<2 \%$ - and we found that the jump is not discontinuous and lower than $-\pi$ rad.

\section{Quantum path interferences}

We have seen in the previous two sections that the HHG phase behavior depends strongly on the type of trajectory (short versus long) and on the value of the electric field at recombination time, thus entangling the structural information contained in the two-center interferences and the electron dynamics.

In practice, the influence of the electron dynamics on a structural interference could be observed by following the phase difference between the short or long trajectory contributions when varying the laser intensity, e.g., by recording

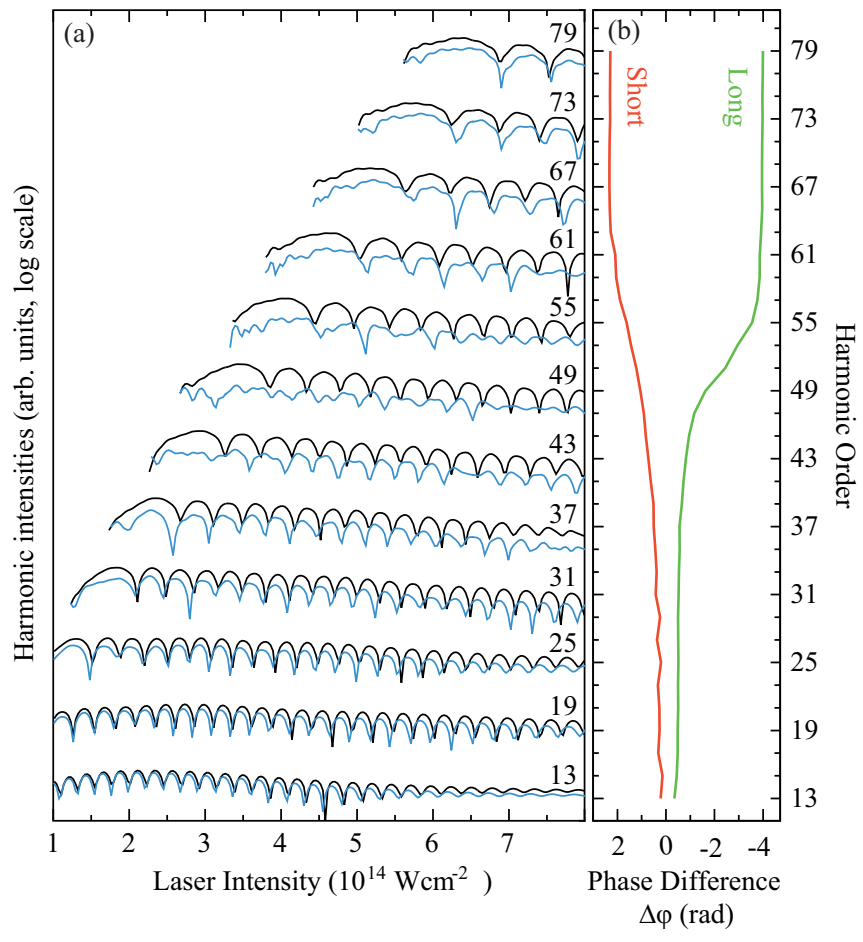

FIG. 8. (a) QPI for the model molecule with $R=1.4$ a.u. (blue lines) and the atomic reference (black lines) from TDSE computations. The curves corresponding to the different harmonic orders are shifted vertically for clarity; (b) phase difference $\Delta \varphi$ between the molecular and the atomic $I_{\mathrm{L}} \rightarrow \alpha$ Fourier transform components of the QPI, associated with the short (red lines) and long (green lines) trajectory contributions.

the quantum path interferences (QPI) in the total harmonic dipole [83,84]. We performed such an analysis in our TDSE simulations, and we show the results in Fig. 8(a) for both the molecule and the reference atom. For each harmonic, we observe oscillations corresponding to the interferences between the short and long trajectories. They mainly come from the phases accumulated by the electron during its excursion in the continuum, i.e., during the second step in HHG, which naturally depends on the trajectory duration. However, for molecules, one also has to take into account the phase of $\widetilde{d}_{\mathrm{rec}}$, which, as seen above, depends significantly on the continuum electron dynamics and is different for each contribution. In fact, as we can see in Fig. 6, for harmonics far from the interference (H49), the recombination dipole phase difference for the two trajectory contributions is either $\approx 0$ or $\approx 2 \pi \mathrm{rad}$, leading to oscillations in phase with the reference atom. For harmonics around $\mathrm{H} 49$, the phase difference is $\approx \pi \mathrm{rad}$ and the QPI exhibit oscillations out of phase with the reference atom. This clear signature makes the QPI a method of choice to observe a pure structural interference modified by electron dynamics.

We extracted these phases by performing an $I_{\mathrm{L}} \rightarrow \alpha$ Fourier transform of the QPI, as described in [85]. We present in Fig. 8(b) the phase difference $\Delta \varphi$ between the model molecule and the reference atom. We observe phase jumps that are an average of the different phase jumps over the laser intensity, and that clearly reproduce the ex- 
pected behavior, i.e., smooth $\pi$ jumps occurring around $\mathrm{H} 49$, in opposite directions for the long and short trajectories, respectively.

Below, we shall show how the Taylor expansions of the molecular SFA times, action, and dipole presented in Sec. II D give a comprehensive understanding of the variations of the HHG dipole phase jump with respect to $R$ and $I_{L}$.

\section{INTERPRETATION}

In this section, we examine the dipole $\widetilde{D}(\Omega)$ given by Eq. (38), investigating each of its terms (see Sec. II D 2). We focus in particular on the role of the modified recombination dipole matrix element $\widetilde{d}_{\text {rec }}$ of Eq. (39).

\section{A. Recombination dipole matrix element}

\section{Explanation of the phase jumps}

We already explained in Sec. III that the zero of $d_{\text {rec }}$ [Eq. (16)] encountered at $p_{0}$, see Eq. (41), encodes the position of the destructive interference. For symmetry reasons (the ground-state wave function is even), this recombination dipole matrix element is a real number in the $\mathrm{PW}$ approximation. Thus, it is constrained to the real axis and its evolution around $p_{0}$ from a positive to a negative value implies a discontinuity of its phase, with a jump of exactly $-\pi$ rad.

However, the modified recombination dipole matrix element $\widetilde{d}_{\text {rec }}$ expressed in Eq. (39) evolves in a totally different manner around $p_{0}$. As it contains a nonzero imaginary part, it is no longer constrained to the real axis and describes a path in the complex plane when varying the harmonic order. It turns out that this imaginary part is proportional to the instantaneous electric field at the recombination time; see Eqs. (33) and (39). Figure 9(a) displays the values of $\widetilde{d}_{\text {rec }}$ in the complex plane for the short trajectories, for different laser intensities, when scanning the harmonic order. The path in the complex plane, followed at a fixed intensity, crosses the $\operatorname{Re}\left(\widetilde{d}_{\text {rec }}\right)=0$ line for a nonzero imaginary part, implying a smooth variation of the phase. In addition, we observe that the higher the electric field at recombination, the further the path is from the origin, and consequently the smoother is the phase jump. Furthermore, as the electric field at the recombination is always positive for the short trajectories, as pointed out in Sec. III, we observe a systematic negative phase jump as shown in Fig. 9(b). This behavior is fully imprinted on the phase of the full HHG dipole as seen in Fig. 7(a). Note that for the lowest intensities, the phase jump is not complete due to the low cutoff value.

The situation is completely different for the long trajectories for which the recombination times are distributed across two laser half-cycles: the electric field is first positive, crosses zero, and is then negative when decreasing the harmonic order. As we can see in Fig. 10(a) for the particular intensity denoted $I_{z}$ (black dashed line), the path followed by $\widetilde{d}_{\text {rec }}$ crosses the origin. This corresponds to a zero electric field at recombination time and thus a zero imaginary part, when the real part also cancels. Therefore, we retrieve the ideal case for which the phase is discontinuous and jumps to $\pm \pi$
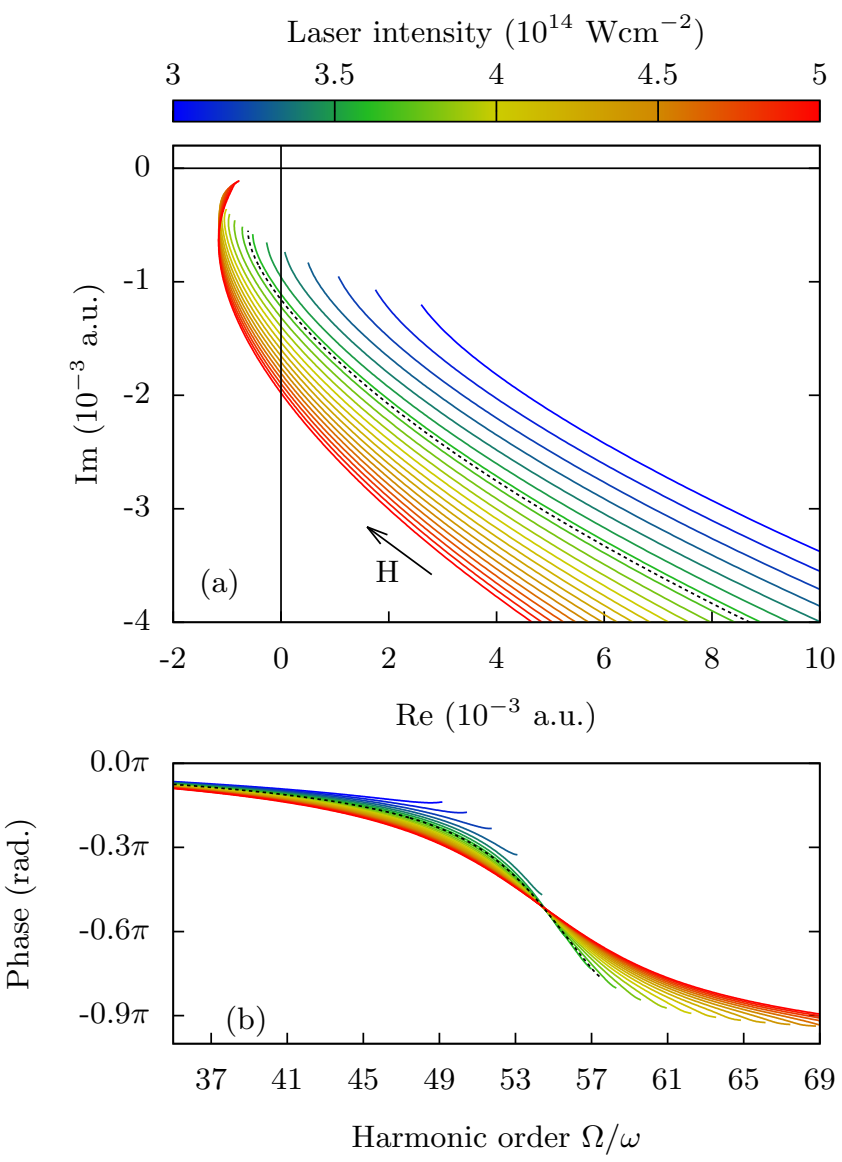

FIG. 9. Modified recombination dipole matrix element $\widetilde{d}_{\text {rec }}$ [Eq. (39)] in the complex plane for the short trajectories (a). The different paths correspond to different laser intensities between 3 and $5 \times 10^{14} \mathrm{~W} \mathrm{~cm}^{-2}$ and for the specific intensity of $I_{z}=3.641 \times 10^{14} \mathrm{~W} \mathrm{~cm}^{-2}$ (black dashed line). Each path shows the evolution of $\widetilde{d}_{\text {rec }}$ with the harmonic order $\mathrm{H}$ (see black arrow). Note that the position of the destructive interference corresponds approximately to the intersection between the curves and the imaginary axis (i.e., zero real part). The phase of $\widetilde{d}_{\text {rec }}$ is reported in (b).

rad [see Fig. 10(b)]. For the other intensities, the laser field is not zero and the phase evolves slowly, varying from zero to $-\pi(\pi) \mathrm{rad}$ for a positive (negative) electric field at the recombination.

Thus, we fully understand the behavior of the recombination dipole matrix element $\widetilde{d}_{\text {rec }}$, which mainly drives the HHG phase evolution along the two-center destructive interference. Its deviations from the ideal case are governed by the continuum dynamics of the electron during the second step of HHG. This leads to a phase jump whose shape depends on the instantaneous value of the electric field at recombination time. Since the electrons following short or long trajectories recombine with the molecule within different time windows, the corresponding phase jumps behave completely differently. As we explained, three different behaviors are notably observed for the long trajectories. We analyze below quantitatively the conditions for which we can observe these three situations. 

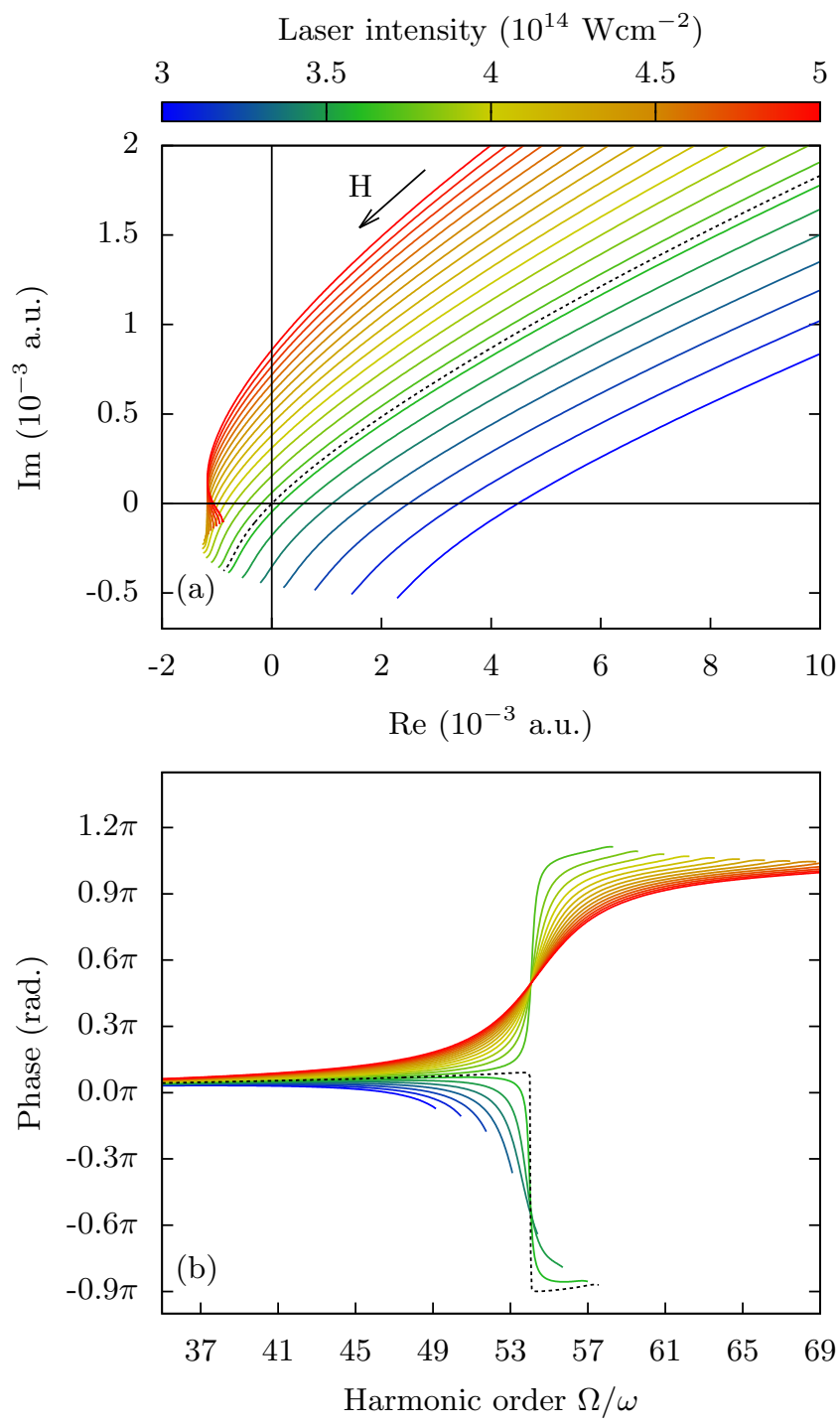

FIG. 10. Same as in Fig. 9 but for the long trajectories. The particular path that crosses the origin corresponds to an intensity of $I_{z}=3.641 \times 10^{14} \mathrm{~W} \mathrm{~cm}^{-2}$ (black dashed line).

\section{Inversion condition}

In this section, we quantify the conditions to observe the inversion in the phase jump for the long trajectories in our simulations either as a function of internuclear distance $R$ (see Fig. 3 in Sec. III A) or as a function of the laser intensity $I_{\mathrm{L}}$ (see Fig. 6 in Sec. III A or Fig. 7 in Sec. III B).

For the long trajectory contributions, the electric field changes sign at a single harmonic frequency denoted $\Omega_{0}$. Hence, harmonics below the cutoff and beyond (below) $\Omega_{0}$ are emitted in the presence of a positive (negative) electric field.

The value of $\Omega_{0}$ can be obtained with atomic classical calculations [58]. We thus solved the Newton equation for an electron freed at ionization time $t^{\prime}$ and brought back to its initial position $x=0$ at recombination time $t$ under the influence of the laser field only. The recollision time for which the laser field is zero is $t_{c}=3 \pi / 2 \omega$ [see Eq. (5)], leading to the corresponding ionization time:

$$
t_{c}^{\prime} \simeq \frac{1}{\left(\frac{3 \pi}{2}-\frac{2}{3 \pi}\right) \omega}
$$

Thus, the corresponding critical harmonic frequency is

$$
\Omega_{0}=I_{\mathrm{p}}+\frac{E_{\mathrm{L}}^{2}}{2 \omega^{2}}\left[\sin \left(\omega t_{c}^{\prime}\right)+1\right]^{2} \approx I_{\mathrm{p}}+2.98 U_{p},
$$

where $U_{p}=E_{\mathrm{L}}^{2} / 4 \omega^{2}=I_{\mathrm{L}} / 4 \omega^{2}$ is the ponderomotive energy.

Therefore, we have two knobs to tune the relative values of $\Omega_{0}^{(\mathrm{PW})}$, Eq. (41), and $\Omega_{0}$, Eq. (44), i.e., the internuclear distance $R$ for the former and the laser intensity for the latter. By varying these knobs, we can put our system in the three cases that we previously discussed: (i) $\Omega_{0}^{(\mathrm{PW})}<\Omega_{0}$, the phase jump for the long trajectories is smoothed and has an opposite direction from that of the short trajectories; (ii) $\Omega_{0}^{(\mathrm{PW})}=\Omega_{0}$, the phase jump for the long trajectories is discontinuous and is equal to $\pm \pi \mathrm{rad}$; (iii) $\Omega_{0}^{(\mathrm{PW})}>\Omega_{0}$, the phase jump for the long trajectories is smoothed and has the same direction as that of the short trajectories.

Furthermore, we found that the equality of $\Omega_{0}^{(\mathrm{PW})}$ and $\Omega_{0}$ can be obtained for an infinity of couples $\left(I_{\mathrm{L}}, R\right)$, approximately verifying the relation

$$
R \simeq 2.58 \frac{\omega}{\sqrt{I_{\mathrm{L}}}}
$$

Finally, we would like to note that, except in our $a b$ initio computations, the entire phase jump is not observed because $\Omega_{0}$ is close to the cutoff frequency, i.e., $I_{\mathrm{p}}+3.17 U_{p}$ [86], as we can see in Figs. 10(a) or 10(b). Unfortunately, one cannot expand the region between these two frequencies by changing the laser intensity since both depend linearly on it.

The SFA-based analysis of $\widetilde{d}_{\text {rec }}$ thus allows us to relate the discontinuous phase to the recombination in the presence of a zero electric field. However, in Sec. III, both for TDSE and molecular SFA computations, we observed that the sharpest phase jump is obtained when the electric field at recombination time is not exactly zero. In the following section, we derive the first-order expansion of the prefactor $C_{\alpha \beta}$ and explain the origin of this shift.

\section{B. Prefactor correction}

When we obtained the first-order expression of the SFA molecular dipole in Sec. II D 2, we neglected the first order of the molecular prefactor $C_{\alpha \beta}$. This first approach allowed us to get a factorized expression for the molecular dipole (38) that led us to important physical insights into the inversion of the $\pi$ jump observed in the HHG phase associated with the long trajectories. However, as mentioned above, discrepancies remain as to the exact values of the electric field for which we observe the phase-jump inversion. To assess the origin of this difference and to go deeper into the analysis, we complete here the analytic derivation of the expression of $C_{\alpha \beta}$ up to the 
first order as

$$
\begin{aligned}
C_{\alpha \beta}\left(t_{\alpha \beta}, t_{\alpha \beta}^{\prime}\right)= & C_{\mathrm{at}}\left(t_{\mathrm{at}}, t_{\mathrm{at}}^{\prime}\right)\left\{1+\frac{R}{2}\left[\frac{(-1)^{\alpha} E\left(t_{\mathrm{at}}^{\prime}\right)}{2\left[p_{\mathrm{at}}+A\left(t_{\mathrm{at}}^{\prime}\right)\right]^{2}}\right.\right. \\
& +\frac{(-1)^{\beta} E\left(t_{\mathrm{at}}\right)}{2\left[p_{\mathrm{at}}+A\left(t_{\mathrm{at}}\right)\right]^{2}} \\
& +\frac{(-1)^{\alpha}}{\left(t_{\mathrm{at}}-t_{\mathrm{at}}^{\prime}\right)\left[p_{\mathrm{at}}+A\left(t_{\mathrm{at}}^{\prime}\right)\right]} \\
& \left.\left.-\frac{(-1)^{\beta}}{\left(t_{\mathrm{at}}-t_{\mathrm{at}}^{\prime}\right)\left[p_{\mathrm{at}}+A\left(t_{\mathrm{at}}\right)\right]}\right]\right\} .
\end{aligned}
$$

For details, see Appendix B.

We then need to sum over the four trajectories (i.e., over $\alpha$ and $\beta$ ) to obtain the full first-order expression of the total dipole (25). The latter can again be factorized in the same form as in the atomic case (7):

$$
\begin{aligned}
D(\Omega)= & C\left(t_{\mathrm{at}}, t_{\mathrm{at}}^{\prime}\right) \widehat{d}_{\mathrm{rec}}\left(p_{\mathrm{at}}+A\left(t_{\mathrm{at}}\right), t_{\mathrm{at}}, t_{\mathrm{at}}^{\prime}\right) \\
& \times \widehat{d}_{\mathrm{ion}}\left(p_{\mathrm{at}}+A\left(t_{\mathrm{at}}^{\prime}\right), t_{\mathrm{at}}, t_{\mathrm{at}}^{\prime}\right) e^{-i S_{\mathrm{at}}}+O\left(R^{2}\right),
\end{aligned}
$$

where the modified recombination $\widehat{d}_{\text {rec }}$ and ionization $\widehat{d}_{\text {ion }}$ dipoles now include contributions from the prefactor expansion. Their expressions read

$$
\begin{aligned}
\widehat{d}_{\text {rec }}\left(p, t, t^{\prime}\right)= & 2 \mathcal{R}(p) \cos \left(\frac{p R}{2}\right) \\
& +2 i \mathcal{Q}\left(p, t, t^{\prime}\right) R \sin \left(\frac{p R}{2}\right),
\end{aligned}
$$

with

$$
\mathcal{Q}\left(p, t, t^{\prime}\right)=-\frac{E(t)}{4} \frac{\partial \bar{\phi}_{s}}{\partial p}(p)-\frac{E(t)}{8 p} \bar{\phi}_{s}(p)-\frac{\bar{\phi}_{s}(p)}{4\left(t-t^{\prime}\right)}
$$

and

$$
\begin{aligned}
\widehat{d}_{\text {ion }}\left(p, t, t^{\prime}\right)= & 2 \mathcal{I}_{\text {at }}\left(p, t^{\prime}\right) \cos \left(\frac{p R}{2}\right) \\
& +2 i \mathcal{K}\left(p, t, t^{\prime}\right) R \sin \left(\frac{p R}{2}\right),
\end{aligned}
$$

with

$$
\begin{aligned}
\mathcal{K}\left(p, t, t^{\prime}\right)= & \frac{E\left(t^{\prime}\right)}{4} \bar{\phi}_{s}(p)-i \frac{E^{2}\left(t^{\prime}\right)}{4 p} \frac{\partial^{2} \bar{\phi}_{s}}{\partial p^{2}}(p) \\
& +i \frac{\partial \bar{\phi}_{s}}{\partial p}(p)\left[\frac{3}{2\left(t-t^{\prime}\right) p}-\frac{E\left(t^{\prime}\right)}{4 I_{\mathrm{p}}}+\frac{\omega^{2} A\left(t^{\prime}\right)}{p}\right] .
\end{aligned}
$$

We thus recover the same kind of expressions as before, with additional terms in the modified recombination $\widehat{d}_{\text {rec }}$ and ionization $\widehat{d}_{\text {ion }}$ dipoles that account for the influence of the prefactor. In fact, these expressions are reminiscent of Eqs. (33) and (36), where $\mathcal{R}^{(1)}$ and $\mathcal{I}^{(1)}$ defined in Eqs. (39) and (40) are now replaced by $\mathcal{K}$ and $\mathcal{Q}$, respectively. Note that these extra terms correspond to the $\zeta$ parameter introduced in Eq. (4) of Ref. [72]. When looking at the expression of $\widehat{d}_{\text {rec }}$, we remark that, because of these new terms, the imaginary part of $\widehat{d}_{\mathrm{rec}}$, which is related to the real part of $\mathcal{Q}$ in Eq. (49), is no longer proportional to the electric field at recombination time. It thus

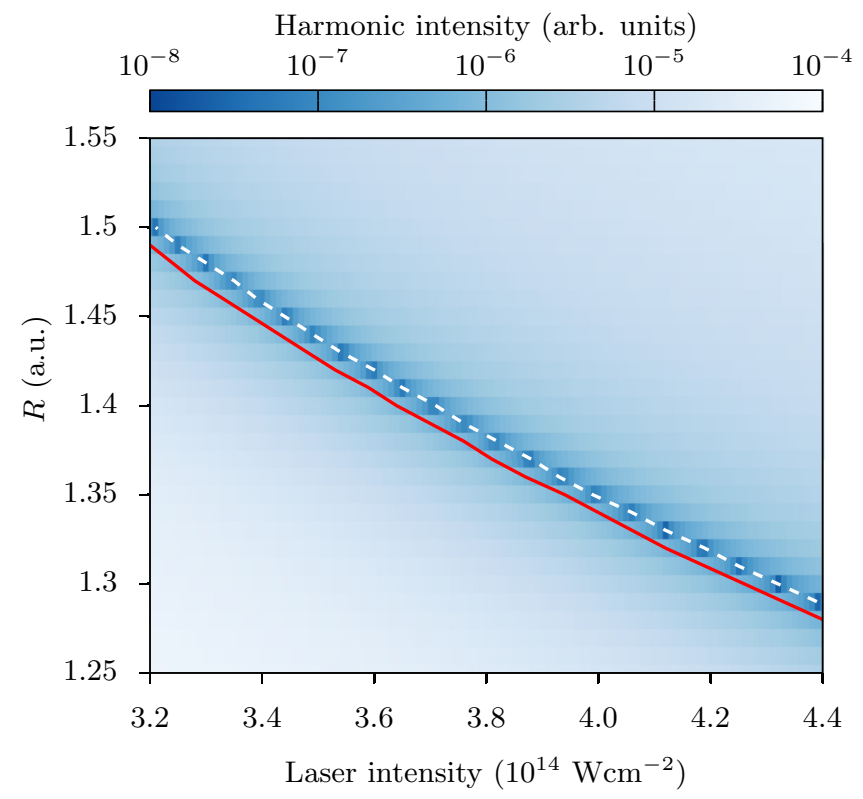

FIG. 11. Value of the minimum of the intensity of the long trajectory contribution in the HHG spectrum as a function of the laser intensity and nuclear distance $R$. The colormap corresponds to the value of the minimum of the molecular SFA dipole Eq. (25). The lines corresponds to the couples $\left(R, I_{\mathrm{L}}\right)$ for which the modified recombination dipole crosses zero in the complex plane: solid red line $\widetilde{d}_{\text {rec }}$, Eq. (39); dotted white line $\widehat{d}_{\text {rec }}$, Eq. (48).

explains why the sharp phase jump occurs for a small nonzero electric field at recombination time.

To better understand the effect of these new terms in the modified recombination dipole, we compare both $\widetilde{d}_{\text {rec }}$ and $\widehat{d}_{\text {rec }}$ to the full SFA results in Fig. 11. The color map indicates the value of the minimum in the full SFA dipole Eq. (25). The darkest pixels correspond to the couples $\left(R, I_{\mathrm{L}}\right)$ for which the minimum is the deepest and the phase jump is the sharpest. The lines show the couples $\left(R, I_{\mathrm{L}}\right)$ for which the modified recombination dipole crosses the origin of the complex plane: the solid red line is for $\widetilde{d}_{\text {rec }}$ (without the prefactor contribution) and the dotted white line is for $\widehat{d}_{\text {rec }}$ (with the prefactor contribution). It is striking that the prefactor contribution allows us to completely recover the correct behavior. Indeed, the zeros of $\widehat{d}_{\text {rec }}$ perfectly match the deep minima in the SFA spectra. As a consequence, the study of the modified recombination dipole $\widehat{d}_{\text {rec }}$ is sufficient to fully recover the position and the shape of both the structural minimum and the phase jump expected in diatomic molecular HHG.

\section{CONCLUSION}

In this paper, we have revisited the phenomenon of twocenter interferences in molecular $\mathrm{HHG}$, with a strong focus on its spectral phase. Using both TDSE and molecular SFA computations, which display similar results, we have shown that the phase for the short and long trajectory contributions behave in totally different ways. In particular, the destructive interference phase jump for the former is always smooth and negative, while for the latter the features of the jump strongly depend on the internuclear distance and on the laser intensity. 
With the help of a time-frequency analysis of the numerical results, we have attributed this effect to the value (magnitude and sign) of the driving electric field when the harmonics are emitted, i.e., at recombination time. We confirmed these findings analytically by performing a Taylor expansion of the molecular SFA around the reference atomic SFA. As was done in $[56,57]$, this expansion allowed us to factorize the SFA dipole expression with a modified recombination dipole matrix element. We found that if we neglect the molecular specificities of the prefactor involved in the factorized expression, then this modified recombination dipole behaves very similarly to the one found in [56,57]: it exhibits a sharp phase jump if and only if the electric field vanishes at the recombination time. However, we showed that this is not in perfect agreement with our TDSE simulations, for which the sharp phase jump appears for a small nonzero electric field at recombination. By consistently taking into account the contribution from the prefactor, our approach allows us to fully explain this behavior.

Our study also confirms that structural two-center interferences can be strongly affected by the instantaneous value of the laser field at recombination and that, in this case, factorization of the HHG dipole proposed in the quantitative rescattering theory [87-89] is not straightforward. More precisely, when one deals explicitly with the two atomic centers of the molecule, this leads to four different classes of electron trajectories, which imprint their signatures inside the recombination dipole matrix element. Thus, the freed electron dynamics and the recombination steps are entangled, and the transition dipole matrix elements cannot be assimilated to that of the static case.

Finally, as the phases of the short and long trajectories evolve differently, an interferometric experimental setup based on quantum path interferences $[83,84]$ should be able to reveal their signatures.

\section{ACKNOWLEDGMENTS}

We acknowledge financial support from the LABEX Plas@Par-ANR-11-IDEX-0004-02 and ANR-10-LABX0039-PALM, the programs ANR11-EQPX0005-ATTOLAB, ANR-15-CE30-0001-01-CIMBAAD, and the European Networks ITN-ATTOFEL and ITN-MEDEA. C.L. acknowledges financial support from the Wiener Wissenschafts- und TechnologieFonds (WWTF) project No. MA16-066 (“SEQUEX”).

\section{APPENDIX A: SECOND-ORDER CORRECTIONS}

From Eqs. (24) and (26), we obtain the following set of equations for the second-order terms:

$$
\begin{aligned}
0= & {\left[p_{\mathrm{at}}+A\left(t_{\mathrm{at}}\right)\right] \Delta t_{\alpha \beta}^{(2)}-\left[p_{\mathrm{at}}+A\left(t_{\mathrm{at}}^{\prime}\right)\right] \Delta t_{\alpha \beta}^{\prime(2)} } \\
& +\left(t_{\mathrm{at}}-t_{\mathrm{at}}^{\prime}\right) \Delta p_{\alpha \beta}^{(2)}-\frac{E\left(t_{\mathrm{at}}\right) R^{2}}{8\left[p_{\mathrm{at}}+A\left(t_{\mathrm{at}}\right)\right]}-\frac{E\left(t_{\mathrm{at}}^{\prime}\right) R^{2}}{8\left[p_{\mathrm{at}}+A\left(t_{\mathrm{at}}^{\prime}\right)\right]}, \\
0= & -\left[p_{\mathrm{at}}+A\left(t_{\mathrm{at}}\right)\right] E\left(t_{\mathrm{at}}\right) \Delta t_{\alpha \beta}^{(2)}+\left[p_{\mathrm{at}}+A\left(t_{\mathrm{at}}\right)\right] \Delta p_{\alpha \beta}^{(2)} \\
& +\frac{E\left(t_{\mathrm{at}}\right)^{2} R^{2}}{16\left(\Omega-I_{\mathrm{p}}\right)}+\frac{\omega^{2} A\left(t_{\mathrm{at}}\right) R^{2}}{8\left[p_{\mathrm{at}}+A\left(t_{\mathrm{at}}\right)\right]},
\end{aligned}
$$

$$
\begin{aligned}
0= & -\left[p_{\mathrm{at}}+A\left(t_{\mathrm{at}}^{\prime}\right)\right] E\left(t_{\mathrm{at}}^{\prime}\right) \Delta t_{\alpha \beta}^{\prime(2)}+\left[p_{\mathrm{at}}+A\left(t_{\mathrm{at}}^{\prime}\right)\right] \Delta p_{\alpha \beta}^{(2)} \\
& -\frac{E\left(t_{\mathrm{at}}^{\prime}\right)^{2} R^{2}}{16 I_{\mathrm{p}}}+\frac{\omega^{2} A\left(t_{\mathrm{at}}^{\prime}\right) R^{2}}{8\left[p_{\mathrm{at}}+A\left(t_{\mathrm{at}}^{\prime}\right)\right]} .
\end{aligned}
$$

That can be inverted to get the second-order terms:

$$
\begin{aligned}
\Delta p_{\alpha \beta}^{(2)}= & \frac{\omega^{2} R^{2}}{8 \Delta}\left(\frac{A\left(t_{\mathrm{at}}^{\prime}\right) E\left(t_{\mathrm{at}}\right)}{p_{\mathrm{at}}+A\left(t_{\mathrm{at}}^{\prime}\right)}-\frac{A\left(t_{\mathrm{at}}\right) E\left(t_{\mathrm{at}}^{\prime}\right)}{p_{\mathrm{at}}+A\left(t_{\mathrm{at}}\right)}\right), \\
\Delta t_{\alpha \beta}^{(2)}= & \frac{E\left(t_{\mathrm{at}}\right) R^{2}}{16\left(\Omega-I_{\mathrm{p}}\right)\left[p_{\mathrm{at}}+A\left(t_{\mathrm{at}}\right)\right]}+\frac{\omega^{2} A\left(t_{\mathrm{at}}^{\prime}\right) R^{2}}{8\left[p_{\mathrm{at}}+A\left(t_{\mathrm{at}}^{\prime}\right)\right] \Delta} \\
& +\frac{\omega^{2} A\left(t_{\mathrm{at}}\right) R^{2}}{16\left(\Omega-I_{\mathrm{p}}\right) \Delta}\left[\left(t_{\mathrm{at}}-t_{\mathrm{at}}^{\prime}\right) E\left(t_{\mathrm{at}}^{\prime}\right)+p_{\mathrm{at}}+A\left(t_{\mathrm{at}}^{\prime}\right)\right], \\
\Delta t_{\alpha \beta}^{\prime(2)}= & -\frac{E\left(t_{\mathrm{at}}^{\prime}\right) R^{2}}{16 I_{\mathrm{p}}\left[p_{\mathrm{at}}+A\left(t_{\mathrm{at}}^{\prime}\right)\right]}-\frac{\omega^{2} A\left(t_{\mathrm{at}}\right) R^{2}}{8\left[p_{\mathrm{at}}+A\left(t_{\mathrm{at}}\right)\right] \Delta} \\
& -\frac{\omega^{2} A\left(t_{\mathrm{at}}^{\prime}\right) R^{2}}{16 I_{\mathrm{p}} \Delta}\left[\left(t_{\mathrm{at}}-t_{\mathrm{at}}^{\prime}\right) E\left(t_{\mathrm{at}}\right)+p_{\mathrm{at}}+A\left(t_{\mathrm{at}}\right)\right] .
\end{aligned}
$$

(A2c)

\section{APPENDIX B: PREFACTOR DERIVATION}

We first use the chain rule for computing the derivative of the composition of functions to find

$$
\begin{aligned}
\frac{\partial^{2} S_{\alpha \beta}^{(p)}}{\partial t^{2}}\left(t, t^{\prime}\right)= & -E(t)\left[p_{\alpha \beta}+A(t)\right]-\frac{\left[p_{\alpha \beta}+A(t)\right]^{2}}{t-t^{\prime}} \\
& +\frac{(-1)^{\beta} R}{2} \omega^{2} A(t), \\
\frac{\partial^{2} S_{\alpha \beta}^{(p)}}{\partial t^{\prime 2}}\left(t, t^{\prime}\right)= & E\left(t^{\prime}\right)\left[p_{\alpha \beta}+A\left(t^{\prime}\right)\right]-\frac{\left[p_{\alpha \beta}+A\left(t^{\prime}\right)\right]^{2}}{t-t^{\prime}} \\
& -\frac{(-1)^{\alpha} R}{2} \omega^{2} A\left(t^{\prime}\right), \\
\frac{\partial^{2} S_{\alpha \beta}^{(p)}}{\partial t \partial t^{\prime}}\left(t, t^{\prime}\right)= & \frac{\left[p_{\alpha \beta}+A(t)\right]\left[p_{\alpha \beta}+A\left(t^{\prime}\right)\right]}{t-t^{\prime}} .
\end{aligned}
$$

We can thus expand the Hessian determinant as

$$
\begin{aligned}
\operatorname{det} S_{\alpha \beta, p}^{\prime \prime}\left(t_{\alpha \beta}, t_{\alpha \beta}^{\prime}\right)= & \operatorname{det} S_{p}^{\prime \prime}\left(t_{\mathrm{at}}, t_{\mathrm{at}}^{\prime}\right)\left\{1-\frac{R}{2}\left[\frac{(-1)^{\alpha} E\left(t_{\mathrm{at}}^{\prime}\right)}{\left[p_{\mathrm{at}}+A\left(t_{\mathrm{at}}^{\prime}\right)\right]^{2}}\right.\right. \\
& +\frac{(-1)^{\beta} E\left(t_{\mathrm{at}}\right)}{\left[p_{\mathrm{at}}+A\left(t_{\mathrm{at}}\right)\right]^{2}} \\
& -\frac{(-1)^{\alpha}}{\left(t_{\mathrm{at}}-t_{\mathrm{at}}^{\prime}\right)\left[p_{\mathrm{at}}+A\left(t_{\mathrm{at}}^{\prime}\right)\right]} \\
& \left.\left.+\frac{(-1)^{\beta}}{\left(t_{\mathrm{at}}-t_{\mathrm{at}}^{\prime}\right)\left[p_{\mathrm{at}}+A\left(t_{\mathrm{at}}\right)\right]}\right]\right\}+O\left(R^{2}\right)
\end{aligned}
$$


[1] A. Scrinzi, M. Y. Ivanov, R. Kienberger, and D. M. Villeneuve, J. Phys. B 39, R1 (2006).

[2] F. Krausz and M. Ivanov, Rev. Mod. Phys. 81, 163 (2009).

[3] G. Farkas and C. Tóth, Phys. Lett. A 168, 447 (1992).

[4] P. Antoine, A. L'Huillier, and M. Lewenstein, Phys. Rev. Lett. 77, 1234 (1996).

[5] P. Antoine, D. B. Milošević, A. L'Huillier, M. B. Gaarde, P. Salières, and M. Lewenstein, Phys. Rev. A 56, 4960 (1997).

[6] I. P. Christov, M. M. Murnane, and H. C. Kapteyn, Phys. Rev. A 57, R2285 (1998).

[7] P. M. Paul, E. S. Toma, P. Breger, G. Mullot, F. Augé, P. Balcou, H. G. Muller, and P. Agostini, Science 292, 1689 (2001).

[8] Y. Mairesse, A. d. Bohan, L. J. Frasinski, H. Merdji, L. C. Dinu, P. Monchicourt, P. Breger, M. Kovačev, R. Taïeb, B. Carré, H. G. Muller, P. Agostini, and P. Salières, Science 302, 1540 (2003).

[9] N. Dudovich, O. Smirnova, J. Levesque, Y. Mairesse, M. Y. Ivanov, D. M. Villeneuve, and P. B. Corkum, Nat. Phys. 2, 781 (2006).

[10] M. B. Gaarde, J. L. Tate, and K. J. Schafer, J. Phys. B 41, 132001 (2008).

[11] A. L. Cavalieri, N. Müller, T. Uphues, V. S. Yakovlev, A. Baltuška, B. Horvath, B. Schmidt, L. Blümel, R. Holzwarth, S. Hendel, M. Drescher, U. Kleineberg, P. M. Echenique, R. Kienberger, F. Krausz, and U. Heinzmann, Nature (London) 449, 1029 (2007).

[12] S. Haessler, B. Fabre, J. Higuet, J. Caillat, T. Ruchon, P. Breger, B. Carré, E. Constant, A. Maquet, E. Mével, P. Salières, R. Taïeb, and Y. Mairesse, Phys. Rev. A 80, 011404 (2009).

[13] K. Varjú, P. Johnsson, J. Mauritsson, A. L'Huillier, and R. López-Martens, Am. J. Phys. 77, 389 (2009).

[14] K. Klünder, J. M. Dahlström, M. Gisselbrecht, T. Fordell, M. Swoboda, D. Guénot, P. Johnsson, J. Caillat, J. Mauritsson, A. Maquet, R. Taïeb, and A. L'Huillier, Phys. Rev. Lett. 106, 143002 (2011).

[15] J. Caillat, A. Maquet, S. Haessler, B. Fabre, T. Ruchon, P. Salières, Y. Mairesse, and R. Taïeb, Phys. Rev. Lett. 106, 093002 (2011).

[16] C. Lévêque, D. Peláez, H. Köppel, and R. Taïeb, Nat. Commun. 5, 4126 (2014).

[17] M. Lein, Phys. Rev. Lett. 94, 053004 (2005).

[18] M. Uiberacker, T. Uphues, M. Schultze, A. J. Verhoef, V. Yakovlev, M. F. Kling, J. Rauschenberger, N. M. Kabachnik, H. Schröder, M. Lezius, K. L. Kompa, H.-G. Muller, M. J. J. Vrakking, S. Hendel, U. Kleineberg, U. Heinzmann, M. Drescher, and F. Krausz, Nature (London) 446, 627 (2007).

[19] S. Baker, J. S. Robinson, C. A. Haworth, H. Teng, R. A. Smith, C. C. Chirilă, M. Lein, J. W. G. Tisch, and J. P. Marangos, Science 312, 424 (2006).

[20] S. Baker, J. S. Robinson, M. Lein, C. C. Chirilă, R. Torres, H. C. Bandulet, D. Comtois, J. C. Kieffer, D. M. Villeneuve, J. W. G. Tisch, and J. P. Marangos, Phys. Rev. Lett. 101, 053901 (2008).

[21] S. Ramakrishna and T. Seideman, Phys. Rev. A 77, 053411 (2008).

[22] S. Haessler, J. Caillat, W. Boutu, C. Giovanetti-Teixeira, T. Ruchon, T. Auguste, Z. Diveki, P. Breger, A. Maquet, B. Carré, R. Taïeb, and P. Salières, Nat. Phys. 6, 200 (2010).

[23] J. Zhao and M. Lein, J. Phys. Chem. A 116, 2723 (2012).
[24] J. Itatani, J. Levesque, D. Zeidler, H. Niikura, H. Pépin, J. C. Kieffer, P. B. Corkum, and D. M. Villeneuve, Nature (London) 432, 867 (2004).

[25] C. Vozzi, M. Negro, F. Calegari, G. Sansone, M. Nisoli, S. De Silvestri, and S. Stagira, Nat. Phys. 7, 822 (2011).

[26] P. Salières, A. Maquet, S. Haessler, J. Caillat, and R. Taïeb, Rep. Prog. Phys. 75, 062401 (2012).

[27] S. Haessler, J. Caillat, and P. Salières, J. Phys. B 44, 203001 (2011).

[28] C. D. Lin, A.-T. Le, Z. Chen, T. Morishita, and R. Lucchese, J. Phys. B 43, 122001 (2010).

[29] V. Véniard, R. Taïeb, and A. Maquet, Phys. Rev. A 54, 721 (1996).

[30] H. G. Muller, Appl. Phys. B 74, s17 (2014).

[31] E. Constant, V. D. Taranukhin, A. Stolow, and P. B. Corkum, Phys. Rev. A 56, 3870 (1997).

[32] J. Itatani, F. Quéré, G. L. Yudin, M. Y. Ivanov, F. Krausz, and P. B. Corkum, Phys. Rev. Lett. 88, 173903 (2002).

[33] O. Smirnova, Y. Mairesse, S. Patchkovskii, N. Dudovich, D. Villeneuve, P. Corkum, and M. Y. Ivanov, Nature (London) 460 , 972 (2009).

[34] X. Zhou, R. Lock, W. Li, N. Wagner, M. M. Murnane, and H. C. Kapteyn, Phys. Rev. Lett. 100, 073902 (2008).

[35] A. Camper, T. Ruchon, D. Gauthier, O. Gobert, P. Salières, B. Carré, and T. Auguste, Phys. Rev. A 89, 043843 (2014).

[36] M. Chini, S. Gilbertson, S. D. Khan, and Z. Chang, Opt. Express 18, 13006 (2010)

[37] A. Camper, Ph.D. thesis, Paris 11 (2014).

[38] B. K. McFarland, J. P. Farrell, P. H. Bucksbaum, and M. Gühr, Phys. Rev. A 80, 033412 (2009).

[39] Y. Mairesse, N. Dudovich, D. Zeidler, M. Spanner, D. M. Villeneuve, and P. B. Corkum, J. Phys. B 43, 065401 (2010).

[40] A.-T. Le, T. Morishita, and C. D. Lin, Phys. Rev. A 78, 023814 (2008).

[41] V. Strelkov, Phys. Rev. Lett. 104, 123901 (2010).

[42] V. V. Strelkov, M. A. Khokhlova, and N. Y. Shubin, Phys. Rev. A 89, 053833 (2014).

[43] J. Rothhardt, S. Hädrich, S. Demmler, M. Krebs, S. Fritzsche, J. Limpert, and A. Tünnermann, Phys. Rev. Lett. 112, 233002 (2014).

[44] M. Kotur, D. Guenot, A. Jimenez-Galan, D. Kroon, E. W. Larsen, M. Louisy, S. Bengtsson, M. Miranda, J. Mauritsson, C. L. Arnold, S. E. Canton, M. Gisselbrecht, T. Carette, J. M. Dahlstrom, E. Lindroth, A. Maquet, L. Argenti, F. Martin, and A. L'Huillier, Nat. Commun. 7, 10566 (2016).

[45] V. Gruson, L. Barreau, A. Jimenez-Galan, F. Risoud, J. Caillat, A. Maquet, B. Carre, F. Lepetit, J. F. Hergott, T. Ruchon, L. Argenti, R. Taïeb, F. Martin, and P. Salieres, Science 354, 734 (2016).

[46] A. Kaldun, A. Blaettermann, V. Stooss, S. Donsa, H. Wei, R. Pazourek, S. Nagele, C. Ott, C. D. Lin, J. Burgdoerfer, and T. Pfeifer, Science 354, 738 (2016).

[47] L. Argenti, A. Jimenez-Galan, J. Caillat, R. Taïeb, A. Maquet, and F. Martin, Phys. Rev. A 95, 043426 (2017).

[48] Y. Mairesse, J. Higuet, N. Dudovich, D. Shafir, B. Fabre, E. Mével, E. Constant, S. Patchkovskii, Z. Walters, M. Y. Ivanov, and O. Smirnova, Phys. Rev. Lett. 104, 213601 (2010).

[49] C. Figueira de Morisson Faria and B. B. Augstein, Phys. Rev. A 81, 043409 (2010). 
[50] Z. Diveki, A. Camper, S. Haessler, T. Auguste, T. Ruchon, B. Carré, P. Salières, R. Guichard, J. Caillat, A. Maquet, and R. Taïeb, New J. Phys. 14, 023062 (2012).

[51] N. Wagner, X. Zhou, R. Lock, W. Li, A. Wüest, M. Murnane, and H. Kapteyn, Phys. Rev. A 76, 061403 (2007).

[52] M. F. Ciappina, C. C. Chirilă, and M. Lein, Phys. Rev. A 75, 043405 (2007).

[53] W. Boutu, S. Haessler, H. Merdji, P. Breger, G. Waters, M. Stankiewicz, L. J. Frasinski, R. Taïeb, J. Caillat, A. Maquet, P. Monchicourt, B. Carre, and P. Salières, Nat. Phys. 4, 545 (2008).

[54] C. C. Chirilă and M. Lein, Phys. Rev. A 80, 013405 (2009).

[55] A. Etches, M. B. Gaarde, and L. B. Madsen, Phys. Rev. A 84, 023418 (2011).

[56] M. D. Śpiewanowski, A. Etches, and L. B. Madsen, Phys. Rev. A 87, 043424 (2013).

[57] M. D. Śpiewanowski and L. B. Madsen, Phys. Rev. A 89, 043407 (2014).

[58] P. B. Corkum, Phys. Rev. Lett. 71, 1994 (1993).

[59] K. J. Schafer, B. Yang, L. F. DiMauro, and K. C. Kulander, Phys. Rev. Lett. 70, 1599 (1993).

[60] M. Lewenstein, P. Balcou, M. Y. Ivanov, A. l'Huillier, and P. B. Corkum, Phys. Rev. A 49, 2117 (1994).

[61] P. Salières, B. Carré, L. L. Déroff, F. Grasbon, G. G. Paulus, H. Walther, R. Kopold, W. Becker, D. B. Milošević, A. Sanpera, and M. Lewenstein, Science 292, 902 (2001).

[62] M. Lewenstein, P. Salières, and A. L'Huillier, Phys. Rev. A 52, 4747 (1995).

[63] D. B. Milošević and W. Becker, Phys. Rev. A 66, 063417 (2002).

[64] M. Lein, N. Hay, R. Velotta, J. P. Marangos, and P. L. Knight, Phys. Rev. Lett. 88, 183903 (2002).

[65] M. Lein, N. Hay, R. Velotta, J. P. Marangos, and P. L. Knight, Phys. Rev. A 66, 023805 (2002).

[66] X. X. Zhou, X. M. Tong, Z. X. Zhao, and C. D. Lin, Phys. Rev. A 72, 033412 (2005).

[67] X. X. Zhou, X. M. Tong, Z. X. Zhao, and C. D. Lin, Phys. Rev. A 71, 061801 (2005).

[68] G. L. Kamta and A. D. Bandrauk, Phys. Rev. A 71, 053407 (2005).

[69] E. V. van der Zwan and M. Lein, Phys. Rev. A 82, 033405 (2010).

[70] C. C. Chirilă and M. Lein, Phys. Rev. A 73, 023410 (2006).
[71] C. Faria, Phys. Rev. A 76, 043407 (2007).

[72] F. Risoud, C. Lévêque, M. Labeye, J. Caillat, A. Maquet, P. Salières, R. Taïeb, and T. Shaaran, Sci. Rep. 7, 17302 (2017).

[73] K. C. Kulander, K. J. Shafer, and J. L. Krause, in Atoms in Intense Laser Fields, edited by M. Gavrila (Academic, New York, 1992).

[74] S. Chelkowski, T. Bredtmann, and A. D. Bandrauk, Phys. Rev. A 88, 033423 (2013).

[75] K. Watanabe, T. Nakayama, and J. Mottl, J. Quant. Spectrosc. Radiat. Transf. 2, 369 (1962).

[76] K. Burnett, V. C. Reed, J. Cooper, and P. L. Knight, Phys. Rev. A 45, 3347 (1992).

[77] Y. Mairesse, A. de Bohan, L. J. Frasinski, H. Merdji, L. C. Dinu, P. Monchicourt, P. Breger, M. Kovačev, T. Auguste, B. Carré, H. G. Muller, P. Agostini, and P. Salières, Phys. Rev. Lett. 93, 163901 (2004).

[78] J. L. Krause, K. J. Schafer, and K. C. Kulander, Phys. Rev. A 45, 4998 (1992).

[79] L. Chipperfield, P. Knight, J. Tisch, and J. Marangos, Opt. Commun. 264, 494 (2006).

[80] M. J. Seaton and G. Peach, Proc. Phys. Soc. 79, 1296 (1962).

[81] M. Labeye, F. Risoud, A. Maquet, J. Caillat, and R. Taïeb, J. Phys. B 51, 094001 (2018).

[82] F. Risoud, J. Caillat, A. Maquet, R. Taïeb, and C. Lévêque, Phys. Rev. A 88, 043415 (2013).

[83] A. Zaïr, M. Holler, A. Guandalini, F. Schapper, J. Biegert, L. Gallmann, U. Keller, A. S. Wyatt, A. Monmayrant, I. A. Walmsley, E. Cormier, T. Auguste, J. P. Caumes, and P. Salières, Phys. Rev. Lett. 100, 143902 (2008).

[84] A. Zaïr, T. Siegel, S. Sukiasyan, F. Risoud, L. Brugnera, C. Hutchison, Z. Diveki, T. Auguste, J. W. Tisch, P. Salières, M. Y. Ivanov, and J. P. Marangos, Chem. Phys. 414, 184 (2013).

[85] P. Balcou, A. S. Dederichs, M. B. Gaarde, and A. L'Huillier, J. Phys. B 32, 2973 (1999).

[86] A. L'Huillier, M. Lewenstein, P. Salières, P. Balcou, M. Y. Ivanov, J. Larsson, and C. G. Wahlström, Phys. Rev. A 48, R3433 (1993).

[87] A.-T. Le, R. D. Picca, P. D. Fainstein, D. A. Telnov, M. Lein, and C. D. Lin, J. Phys. B 41, 081002 (2008).

[88] A.-T. Le, R. R. Lucchese, S. Tonzani, T. Morishita, and C. D. Lin, Phys. Rev. A 80, 013401 (2009).

[89] A.-T. Le, R. R. Lucchese, and C. D. Lin, Phys. Rev. A 87, 063406 (2013). 